\title{
Usuelle Wortverbindungen in schulischen Kontexten
}

\author{
Katrin Hee (Köln)
}

\begin{abstract}
The language of schooling is characterized by (formulaic) typical linguistic patterns. The existing research on language acquisition assumes that those patterns are acquired implicitely during primary language acquisition without didactical support. This paper questions this presupposition and investigates the mode of pattern acquisition, i.e. whether patterns are indeed acquired as fixed patterns/idiomatic expressions or are rather learned during the development of the language of schooling.
\end{abstract}

\section{$1 \quad$ Einleitung}

Verfestigte Muster ${ }^{1}$ sind v. a. in Institutionen häufig zu finden. In der Institution Schule herrscht neben typischen Interaktions-Mustern wie dem IRE-Schema (u. a. Richert 2005) v. a. ein Sprachgebrauch vor, der an der „konzeptionellen Schriftlichkeit“ (Koch/Oesterreicher 1986) orientiert ist (u. a. Vollmer/Thürmann 2010). Dieser ist geprägt durch spezifische diskursive, syntaktische und lexikalisch-semantische Strukturen (u. a. Gogolin/Lange 2010). In der Literatur wird davon ausgegangen, dass diese von Muttersprachlern implizit erworben werden und keiner Didaktisierung bedürfen. Der Beitrag möchte vor diesem Hintergrund der Frage nach dem Erwerb dieser Struktur- und Ausdrucksformen nachgehen: Werden sie tatsächlich als verfestigte Strukturen erworben oder lassen sich - z. B. mit Blick auf Kollokationen - „Fehler“ resp. nicht-usuelle Verwendungen sowie alternative Formulierungen im aktualgenetischen Erwerb feststellen, die aufzeigen, dass es sich eben gerade nicht um sprachliche Verfestigungen in dem Sinne handelt, dass sie als feststehende sprachliche Ausdrücke erlernt und direkt abgerufen werden, sondern im schulischen Kontext erworben werden?

Der vorliegende Beitrag geht dieser Frage auf Basis eines videobasierten, transkribierten Korpus` von problemlösenden Gruppenunterrichtsphasen (GrU) mit vorausgehenden und daran anschließenden Interaktionen im Plenum (PIU) einer 5., 8. und 11. Gymnasialklasse in den Fächern Deutsch, Mathematik und Geschichte nach. Der methodische Zugang sind Analysen von

\footnotetext{
${ }^{1}$ Vgl. ausführlich zum Konzept der Musterhaftigkeit Steyer (2013: 39-50), die zwischen „Wortverbindungen“ und „Wortverbindungsmuster[n]“ als „mehrgliedrige, lexikalisch teilspezifizierte Einheiten, die feste lexikalische Leerstellen (Slots) enthalten [...] [, wobei die] Slots [...] mit variablen lexikalischen Füllern besetzt [werden]“ (Steyer 2013:338) unterscheidet. Wortverbindungsmuster sind dabei „'semantische und pragmatische Zwitter““, da in „ihnen [...] immer Spuren der Einzelbedeutungen ihrer Wortverbindungskinder eingegraben [sind]“ (Steyer 2013: 345). Zur Auseinandersetzung mit „,mehr oder weniger fest[en] [Wortverbindungen]“ (Steyer Hrsg. 2003) vgl. Stein (2003) zu Formelhaftigkeit und Routinen, Ágel (2004) zu Phraseologismen. Ebenfalls zur Phraseologie vgl. ausführlich Feilke (2007) sowie zu pragmatischen Phraseologismen Beckmann/König (2002). Steyer (2013) beschäftigt sich aus korpuslinguistischer Sicht mit „usuellen Wortverbindungen“.
} 
Interaktionen in Gruppendiskussionen, in denen Schülerinnen und Schüler usuelle Wortverbindungen verwenden und/oder sprachlich an ihnen arbeiten und die so Einblicke in aktualgenetische Erwerbsprozesse erlauben. Fokussiert werden dabei Kollokationen und Funktionsverbgefüge, beides auch mit Blick auf „Vor- und Übergangsformen“ (,,intermediäre Erwerbsformate“; Pohl 2016: 67). ${ }^{2}$ Dabei soll der Blick auf mögliche ungewöhnliche oder nicht-usuelle Kombinationen sowie Formulierungsalternativen erste Hinweise darauf geben, inwieweit es sich bei diesen Strukturen tatsächlich um feste im mentalen Lexikon als solche abrufbare sowie ad-hoc produzierbare Wortverbindungen handelt oder inwieweit sie ggf. vielmehr in der schulischen Sozialisation als solche erworben und routinisiert werden müssen.

\section{Theoretische Grundlagen}

\subsection{Der Unterrichtsdiskurs}

Schulische Interaktion kann systematisch betrachtet als Vier-Felder-Schema dargestellt werden, das sich durch die Kreuzklassifikation von medialer Mündlichkeit und Schriftlichkeit sowie rezeptivem und produktivem Verhalten der Schülerinnen und Schüler (SuS) ergibt (Pohl 2016).

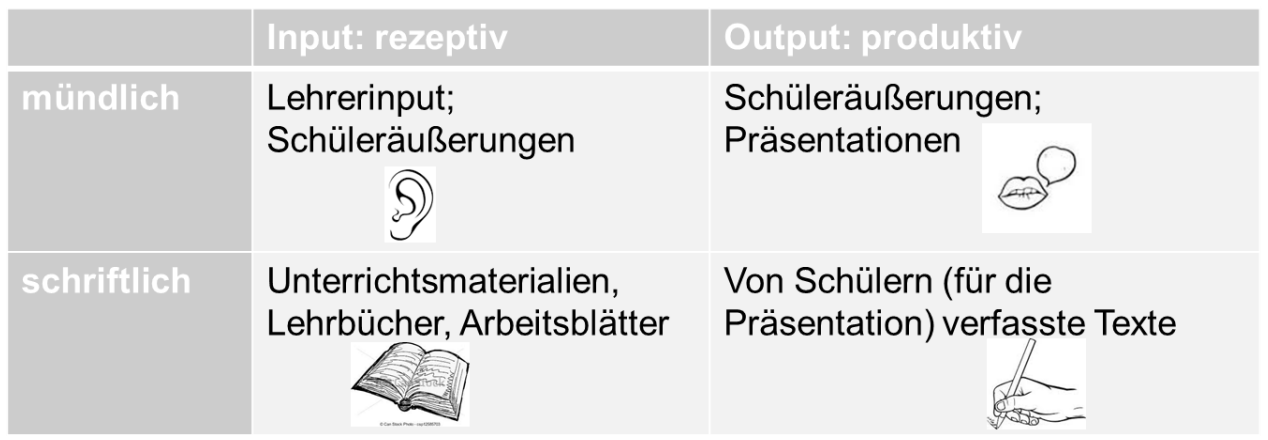

Abbildung 1: Vier-Felder-Schema des Unterrichtsdiskurses (Pohl 2016; modifiziert durch K.H.)

Beispielsweise sind der Lehrerinput oder Äußerungen anderer Schüler medial mündlich, d. h. gesprochen, und für die SuS ein Input, den sie rezeptiv verarbeiten müssen. Die von den SuS verfassten Texte dagegen sind medial schriftlicher Output, d. h. sprachliche Produkte, die sie selbst hergestellt haben. Die vier Felder lassen sich dabei den primärsprachlichen Handlungskompetenzen der Schüler zuordnen, nämlich: zuhören, sprechen, lesen und schreiben.

Diese Systematisierung bezieht sich allerdings lediglich auf die mediale Seite des Unterrichtsdiskurses. Nimmt man darüber hinaus auch die konzeptionelle Dimension mit in den Blick, kann man u. a. mit Quehl (2012: 29) davon ausgehen, dass der Unterrichtsdiskurs in seiner institutionellen Form durchweg konzeptionell schriftlich geprägt ist, d. h. auch in den Feldern, die oben als mündlich ausgewiesen sind: „Die Sprache im Unterricht ist konzeptionell eher

2 Zum Erwerb von Kollokationen in der Fremdsprache cf. ausführlich Wallner (2014). 
schriftlich, und zwar in geschriebener wie in gesprochener Form“" (Dehn 2011: 138). ${ }^{3}$ Der Unterrichtsdiskurs ist dabei auf mehreren Ebenen durch verfestigte ${ }^{4}$ Muster geprägt. Dies können einerseits typische Interaktionsmuster wie das IRE-Schema sein; andererseits fällt darunter auch ein Sprachgebrauch, der an der konzeptionellen Schriftlichkeit (Koch/Oesterreicher 1986) orientiert ist sowie fachsprachlich affiziert ist (u. a. Vollmer/Thürmann 2010) und der entsprechend durch spezifische diskursive, syntaktische und lexikalisch-semantische Strukturen (u. a. Gogolin/Lange 2010) geprägt ist. Dazu gehören unter anderem spezifische Kollokationen (Ahrenholz 2010: 16, Grießhaber 2010: 39) und Funktionsverbgefüge (Roelcke 2002: 17; Czicza/Hennig 2011: 2; Graefen 2000: 6; Wieler 2011; Gogolin/Lange 2011: 114) sowie Wortbildungsmuster (Roelcke 2002: 17; Czicza/Hennig 201: 2; Gogolin/Lange 2011: 114), ${ }^{5}$ die im Folgenden fokussiert werden sollen.

\subsection{Usuelle Wortverbindungen in schulischen Kontexten}

Auf der lexikalisch(-semantisch)en Ebene können mit Blick auf „Sprachliche Verfestigungen“ resp. „sprachlich Verfestigtes“ unterschiedliche Strukturtypen relevant sein.

Das „Miteinandervorkommen von Wörtern“ (Reder 2006: 17) ist in unterschiedlichen linguistischen Ansätzen zu erfassen versucht worden und entsprechend mit unterschiedlichen Begriffen wie „Kollokation“, „,usuelle Wortverbindung“ etc. bezeichnet worden. Auch eine linguistische Abgrenzung ist bisher nicht einheitlich vorgenommen worden. So gibt es Reder (2006: 43) zufolge zwei Modelle, wie Kollokationen von freien Wortverbindungen und Phraseologismen abzugrenzen sind. Dabei werden im ersten Modell Kollokationen als kohyponym zu freien Wortverbindungen und Phraseologismen verstanden, während im zweiten Modell freie Wortverbindungen und Phraseologismen als lediglich zwei Kohyponyme unterschieden und Phraseologismen als Hyperonym von Kollokationen und anderen festen Wortverbindungen verstanden werden (ebd.). ${ }^{6}$

In jüngerer Zeit ist mit dem Modell ,Usuelle Wortverbindungen“ versucht worden, eine übergeordnete Kategorie zu bezeichnen, die ,zunächst allen Mehrwortkandidaten eine Heimat

\footnotetext{
${ }^{3}$ Konzeptionelle Schriftlichkeit steht dabei im Zusammenhang mit Fachsprache, Bildungssprache, Schulsprache etc., worauf hier aber aus Platzgründen nicht näher eingegangen werden kann. Beschäftigt man sich mit der in der Schule gesprochenen Sprache, so findet man in der Literatur häufig den Begriff Bildungssprache.

Mir scheint der Begriff nicht zielführend, da er erstens weder hinreichend empirisch untersucht (vgl. auch Morek/Heller 2012: 90) noch theoretisch ausgearbeitet ist und das Konzept an sich beispielsweise auch keine Darstellung von Entwicklungsverläufen erlaubt, da er keine Zwischenstufen abbilden kann. Es kann hier keine theoretische Begriffsbestimmung erfolgen. Vielmehr soll an dieser Stelle der Kern dieser Konzepte betont werden: Bei allen - auch dem Begriff Bildungssprache - geht es letztendlich um eine an konzeptioneller Schriftlichkeit orientierte Sprachnorm. Vgl. zur Kritik an dem Begriff Bildungssprache u. a. auch Pohl (2016: 59).

${ }^{4}$ Es wird hier von einer „relativen Festigkeit“ ausgegangen, die sich u. a. daran zeigt, dass die Strukturen auch „abweichend von der üblichen Kombination verwendet“" werden können (Margewitsch 2005:188).

${ }^{5}$ Wortbildungen werden hier mitbehandelt, da sie in einer engen Nähe zu Kollokation stehen und teilweise aus ihnen hervorgebracht werden (vgl. Ulrich 2016: 21; Barz 1996, die allerdings auch auf Unterschiede hinweist). Entsprechend führen Häcki Buhofer et al. (2014) in ihrem Kollokationswörterbuch neben Adjektiven/Adverbien, Verben und Nomen auch Zusammensetzungen.

${ }^{6}$ Nach Reder (2006: 44) liegt der Unterscheidung, Kollokationen als eigene oder Unterkategorie von Phraseologismen zu verstehen, die „Frage nach einer, engen“ oder, weitere“ Phraseologismen-Auffassung zu Grunde“.
}

ISSN 1615-3014 
[gibt]“ (Steyer 2013: 14) und „die lexikalische Verfestigung jedweder Mehrworteinheiten aufgrund massenhaften Gebrauchs durch mehrere Sprecher, so wie sie durch korpusanalytische Verfahren rekonstruierbar ist", fokussiert (Steyer 2013: 337).

Augst (demn.) unterscheidet für den Erwerb „usueller Wortverbindungen“ (in Anlehnung an Steyer 2013) in DaF-Kontexten folgende Untertypen: „Schemata“, die er unterteilt in „Komplementärformeln“ und „Schemata vom Typ X ist X“, „Präposition + Substantiv“, „, feste präpositionale Valenz“, „Nominationsstereotype[.]“, „Funktionsverbgefüge“, unterteilt in solche mit nur einer und solche mit mehreren Verbindungen, wobei erstere laut Augst auch als Kollokationen angesehen werden können, „,idiomatische Wendungen“/,Zwillingsformeln“/,WieVergleiche“, die er wegen ihrer funktionalen Ähnlichkeit zusammen fasst, „Kollokationen“, „lexikalische Solidaritäten“, „Routineformeln“, „Formulierungsroutinen“, „Sprichwörter“ sowie ,idiomatische Redewendungen“.

Für den vorliegenden Kontext sind sicherlich die Funktionsverbgefüge und Kollokationen am interessantesten: Sie gehören durch ihre schriftsprachliche Geprägtheit (u. a. Ulrich 2016: 24) zu den für den schulischen Erfolg notwendigen und in der Schule gebrauchten Struktur- und Ausdrucksmitteln, mit denen ,ansprechende, abwechslungsreiche und richtige Texte“ (Häcki Buhofer et al. 2014: X) verfasst werden können. Was unter Kollokation zu verstehen ist, ist allerdings in der linguistischen Forschung bislang nicht einheitlich definiert (Wallner 2014: 13) - auch Augst fasst die Kollokationen einerseits als eigenen Untertyp, andererseits verweist er bei den Funktionsverbgefügen mit nur einer möglichen Verbindung darauf, dass „man sie auch als Kollokationen ansehen [kann]“. ${ }^{7}$ Steyer (2013: 36) versteht Kollokation als ,primär semantisch-konzeptuell motivierte Basis-Kollokator-Dichotomie“. Wallner (2014: 53) bestimmt Kollokationen semantisch als ,schwach bzw. nichtidiomatische Wortverbindungen [...], die mithilfe der Bedeutungen der Bestandteile semantisch weitestgehend erschlossen werden können." Strukturell definiert sie Kollokationen als „binär strukturiert[.]“ (Wallner 2014: 55), wobei die syntaktischen Relationen festgelegt seien, z. B. liege bei den „,verbnominalen Kollokationen des Typs V+S stets eine Verb-Objekt-Beziehung zugrunde“ (Wallner 2014: 55). Weitere Merkmale für das Vorliegen einer Kollokation seien:

1. semantische Abhängigkeit des Kollokators,

2. normbedingte Einschränkungen hinsichtlich der Substituierbarkeit der Bestandteile durch bedeutungsähnliche lexikalische Einheiten,

3. normbedingte morphosyntaktische Transformationskriterien,

4. normbedingte Einschränkungen hinsichtlich der Passivierung sowie in Bezug auf die Pronominalisierung und Erfragung des Objekts bei verbnominalen Kollokationen des Typs $\mathrm{V}+\mathrm{Sa}$,

5. die korpusstatistisch berechnete Assoziation fällt vergleichsweise hoch aus.

Wallner grenzt Kollokationen von Wortidiomen, Funktionsverbgefügen sowie freien Wortverbindungen ab. ${ }^{8}$ Wortidiome versteht sie dabei als ,feste Wortverbindungen [...], die sich durch

\footnotetext{
${ }^{7}$ Auch Feilke (2007: 68) unterscheidet bei den Funktionsverbgefügen (FVG) ,sogenannte Nominalisierungsverbgefüge wie $j m d$. Hilfe leisten, jmd. Antwort geben und im weiteren Substantiv-Verb-Kollokationen (SVKs) wie die Initiative ergreifen".

${ }^{8}$ Ulrich (2016: 20) grenzt sie von freien und festen Wortverbindungen ab und bezeichnet sie als ,halbfeste Wortverbindung“, Häcki Buhofer et al. (2014: XI) bezeichnen sie hingegen als „feste Wortverbindungen“ und grenzen
}

ISSN 1615-3014 
vollständige bzw. teilweise Idiomatizität auszeichnen“ (Wallner 2014: 33), wobei ein ,wesentliches Kennzeichen für das Vorliegen der Idiomatizität ist, dass die Bedeutung mindestens einer Komponente eine Diskrepanz zu allen üblicherweise mit dieser Komponente verbundenen Lesarten aufweist in der wortverbindungsinhärenten Lesart nahezu [...] unikal ist" (Wallner 2014: 39). Funktionsverbgefüge (FVG) grenzt Wallner dahingehend von Kollokationen ab, dass „Verb und Substantiv als eine Einheit das Prädikat bilden“, während es sich bei „Kollokationen mit formal gleicher Struktur i. d. R. um eine Verb-Objekt-Beziehung“" handele (ebd.). Die Abgrenzung zu den freien Wortverbindungen ist laut Wallner (2014: 53) besonders schwierig:

Dass eine Kollokation (statt einer freien Wortverbindung) vorliegt, kann daher nur auf Grundlage von subjektiven Entscheidungen hinsichtlich des Zutreffens eines oder mehrerer der folgenden Merkmale angenommen werden:

1. Die Semantisierung des mutmaßlichen Kollokators kann nur unter Rückgriff auf die Basis erfolgen (semantische Abhängigkeit des Kollokators von der Basis).

2. Die Bestandteile der Wortverbindung sind nur eingeschränkt bzw. überhaupt nicht durch Synonyme substituierbar.

3. Es sind morphosyntaktische Transformationsrestriktionen erkennbar.

4. Die statistisch berechnete Assoziation zwischen den Bestandteilen einer Wortverbindung fällt vergleichsweise hoch aus.

Köster und Neubauer (2002: 286) unterscheiden verschiedene Strukturtypen, bei denen die Basis (fett markiert) jeweils eine andere sein kann:

Adjektiv und Substantiv

Verb + Substantiv

Substantiv + Verb

Substantiv + Substantiv

Adverb + Adjektiv

Verb + Adverb ein schwerer Irrtum

ein Irrtum unterlaufen

Holz spalten

ein Rudel Wölfe

unglücklich verliebt

herzlich lachen

Nach Ulrich (2016:22) weisen Kollokationen

eine nicht symmetrische Struktur auf. Ein Bestandteil dominiert den anderen. Er ist die Basis der Kollokation und wird unabhängig von ihr als selbständiges Lexem gelernt und gespeichert (z. B. der Tisch). Der andere Teil ist der Kollokator (z. B. decken oder abräumen). Er wird beim Formulieren in Abhängigkeit von der Kollokationsbasis gewählt. Die Abfolge beim Formulieren geht immer diesen Weg: von der Basis zum Kollokator, d. h. in den meisten Fällen von einem Substantiv zu einem Verb [...] oder von einem Substantiv zu einem Adjektiv [...]. Kenntnis des passenden Kollokators beschleunigt und optimiert die Formulierung, Unkenntnis führt zu Verlegenheitsformulierungen $[\ldots]$.

Reder (2006: 18f.) unterscheidet bei der Deutung des Kollokationsbegriffes drei Ebenen, nämlich eine abstrakte, eine konkrete und eine kognitive. Hinter der abstrakten Verwendungsweise

sie von „freien Wortverbindungen“ und ,idiomatischen Wortverbindungen/Phrasen“ ab, wobei sie jeweils einen Übergangsbereich skizieren. Köhler (1985) grenzt Funktionsverbgefüge von Festen Fügungen mit Supplementverben ab, die m. E. mit Kollokationen gleichzusetzen sind. 
des Kollokationsbegriffes steht die Idee einer „syntagmatischen Verträglichkeitsbeziehung“9 (Reder 2006: 18) bzw. einer „horizontale[n] Bedeutungsbeziehung“ (Reder 2006: 19), die sich durch häufiges gemeinsames Vorkommen der beiden lexikalischen Elemente ergibt. Die konkrete Beschreibung von Kollokationen erfolgt vor dem Hintergrund des Verständnisses von Kollokation als „polylexikalische Einheit des Sprachsystems“ (Reder 2006: 19). Schließlich wird unter der kognitiven Dimension davon ausgegangen, dass das ,gemeinsame Auftreten von lexikalischen Elementen das mentale Lexikon strukturiert“ (Reder 2006: 19).

Von Kollokationen werden in der Literatur u. a. häufig Funktionsverbgefüge abgegrenzt. Nach Hoffmann (2013: 246) sind Funktionsverbgefüge ${ }^{10}$ Konstruktionen, bei denen sich Verben ,mit nominalisierten Verben oder Adjektiven verbinden“, die die „Kernbedeutung des Prädikatsausdrucks“ ausmachen, während die Funktionsverben ,semantisch ,ausgebleicht““ (Zifonun et al. 1997: 703) sind und „,in dieser Hinsicht den Kopulaverben [gleichen]“ (Zifonun et al. 1997: 704). Entsprechend liegt die eigentliche Bedeutung des FVG im nominalen Teil (vgl. u. a. Eisenberg 2006: 312), d. h. das „Verbalsubstantiv [bildet] den sachverhaltsbeschreibenden Kern, während das Funktionsverb als Träger der verbalen Morphologie allgemeinere verbale Bedeutungsaspekte ausdrückt" (Fabricius-Hansen 2009: 418). Die häufigsten Funktionsverben sind nach Eisenberg (2006: 310) „,kommen“ und „,bringen“, gefolgt von „stehen“, ,geraten“, ,setzen“, „stellen“, „halten“, „nehmen“ (Herv. getilgt, K.H.). Die als ,Nominalstil“ oder ,Bürokratendeutsch“ stigmatisierten FVG (Hoffmann 2013: 247) haben häufig eine Entsprechung als „einfaches Verb“ (Hoffmann 2013: 247) wie zum Beispiel „widersprechen“ vs. „Widerspruch einlegen“ (Hoffmann 2013: 247) oder „abschließen“ vs. „zum Abschluss bringen“ (Eisenberg 2009: 311, Herv. getilgt K.H.), wobei sich ihre Verwendung nach Textsorte unterscheidet: ${ }^{11}$ FVG kommen im Vergleich zu den einfachen Verben gehäuft in Bereichen vor, ,in denen man generell zum Nominalstil“" (Fabricius-Hansen 2009: 425) und damit zu konzeptioneller Schriftlichkeit tendiert, was für den schulischen Bereich der Fall ist (u. a. Lange 2012: 127). FVG können in „kausative“ („Der Kunde gibt eine neue Serie in Auftrag“), „,inchoative“ („Endlich geht das Buch in Druck“), ,durative“" (,Das neue Modell ist bereits in Arbeit“) und ,passivische“ („Das Buch ist noch im Druck“) unterteilt werden (Zifonun et al. 1997: 704), wobei

\footnotetext{
${ }^{9}$ Coseriu erklärt die ,lexikalischen Solidaritäten als syntagmatische Erscheinungsformen, die paradigmatisch bedingt sind (Coseriu 1967: 297)“ (zit. nach Reder 2006: 29), wobei er bei der paradigmatischen Ebene von Wortfeldern ausgeht, aus denen jeweils Elemente ausgewählt werden, die in syntagmatischer Hinsicht miteinander eine Beziehung eingehen, wie „Baum“ aus dem Wortfeld „Pflanzen“ und „fällen“ aus dem Wortfeld „entfernen“.

${ }^{10}$ Hoffmann unterscheidet Funktionsverbgefüge (FVG) von Streckverbgefügen (SVG), ohne sie scharf voneinander abzugrenzen. So ließe sich bei SVG der nominale Teil „oft in den Plural setzen“, auch könne „meist kein eingesetzt werden“ und schließlich sei „eine Adjektiverweiterung [...] meist unproblematisch“ (Hoffmann 2013: 249, fett gesetzte Herv. v. K.H.). Die Abgrenzungsmerkmale sind also sämtlich abgefedert. Betrachtet man nun die für die FVG und SVG aufgeführten Beispiele, zeigt sich, dass diese weich formulierten Kriterien nicht für die aufgeführten Beispiele anwendbar sind: So ist das als FVG aufgeführte Beispiele „,Beachtung finden “ (ebd.: 248) durch „kein“ sowie mit einem Adjektiv wie z. B. „große“ erweiterbar, während beispielsweise die als SVG genannten Beispiele „Widerstand leisten “ oder „Beitrag leisten“ (ebd.: 249) nicht in den Plural gesetzt werden können. Vor diesem Hintergrund wird eine Unterscheidung in SVG und FVG in diesem Beitrag nicht weiter verfolgt.

${ }^{11}$ Es ist an dieser Stelle wichtig darauf hinzuweisen, dass erstens ein ,,solcher Bezug von FVG auf einfache Verben [...] aber nur zum Teil möglich [ist]“ Eisenberg (2006: 311), wie Beispiele wie ,zur Deckung bringen“ oder ,,vor Augen kommen“" (ebd.) belegen, für die kein Bezug zu entsprechenden einfachen Verben hergestellt werden kann. Zweitens sind auch die sich entsprechenden FVG und einfachen Verben nicht synonym und semantisch gleichzusetzen, da sie jeweils eine leicht andere Konnotation haben (Hoffmann 2013: 247; Fabricius-Hansen 2009: 425).
}

ISSN 1615-3014 
letztere „streng genommen keine eigene Klasse“ bilden, sondern kausative, inchoative oder durative FGV mit passivischer Bedeutung sind (ebd.). Die verschiedenen Klassen der FVG geben bereits einen Hinweis auf den Bedeutungsbeitrag der FVG im Vergleich zu den einfachen Verben. Sie erlauben u. a. „besondere Thema-Rhema-Strukturen“, „Passivumschreibungen“, eine „Kausativierung und [...] Signalisierung von Aktionsarten“ (Eisenberg 2002: 316; vgl. auch Fabricius-Hansen 2009: 425, Zifonun et al. 1997: 704f.).

In diesem Zusammenhang relevant erscheint auch das Konzept der „Formative grammatischer Inkongruenz" (Pohl 2007: 417) bzw. der ,grammatical metaphors' (Pohl 2007: 417), in dem „die Wortklasse eine spezifische grammatische Bedeutung einbringt“ (Pohl 2007: 419). Entsprechend deuten $u$. a. Nominalisierungen und komplexe Substantivgruppen auf eine andere semantische Qualität hin als bspw. ihre verbalen Entsprechungen, indem sie in kommunikativer Hinsicht u. a. eine Komprimierungs- und Kondensationsfunktion, in kognitiver Hinsicht $u$. a. eine Abstraktionsfunktion haben (Pohl 2007: 426) und damit im Vergleich zu ihren verbalen Entsprechungen stärker zum Pol konzeptioneller Schriftlichkeit nach Koch und Oesterreicher (u. a. 1986) rücken. „Freie Wortverbindung, Kollokation und komplexes Wort sind demnach drei unterschiedlich [sic!] Ausdrucksformen, die dem Sprecher oder Schreiber wahlweise für die Verbalisierung eines Konzepts zur Verfügung stehen: z. B. Wanderung bei Nacht - nächtliche Wanderung - Nachtwanderung." (Ulrich 2016: 21). ${ }^{12}$ Für den vorliegenden Kontext ist es daher interessant zu schauen, auf welche Variante die SuS jeweils zurückgreifen.

„Eine weitere Annahme geht davon aus, dass die Sprachträger Kollokationen als Einheiten so wie Wörter - kennen. Folglich sind Kollokationen im mentalen Lexikon ähnlich wie ein Wort als Einheit gespeichert und werden nicht aus den Bestandteilen produziert, sondern als Einheit gespeichert und abgerufen" (Reder 2006: 55f., vgl. auch Steyer 2013: 23). Entsprechend gibt es m.W. bisher weitestgehend didaktische Arbeiten zum Erwerb von Kollokationen in der Fremdsprachendidaktik, ${ }^{13}$ in der davon ausgegangen wird, dass die Lerner das mentale Lexikon über das Bewusstwerden von Interferenzen aus ihrer L1 sowie gezieltes Anreichern des jeweiligen sprachlichen Lexems erwerben (Reeder 2006). ${ }^{14}$ Für muttersprachliche Lerner wird hingegen davon ausgegangen, dass das mentale Lexikon die entsprechenden Informationen bezüglich Kollokationen bereit hält, die Sprecher also direkten Zugriff darauf haben und sie nicht erst erwerben müssen (Reder 2006: 55f., vgl. auch Steyer 2013:23). Der folgende Beitrag geht hingegen von der Annahme aus, dass in schulischen Kontexten gebräuchliche Kollokationen von den SuS nicht im primären Spracherwerb, sondern erst schulisch flankiert erworben werden müssen, da sie als sog. ,bildungssprachliche' Strukturen/Merkmale im natürlichen Spracherwerb der SuS nicht dominant auftreten, sondern erst im ,Lebensraum Schule` (Cathomas 2007: 180) relevant werden. Dabei wird von der Annahme ausgegangen, dass sie nicht adhoc als sprachliche Verfestigungen erworben werden, sondern die Schülerinnen und Schüler sie

\footnotetext{
${ }^{12}$ Für die „Nähe von Wortbildung und Kollokation“, die sich unter anderem auch darin zeigt, ,dass es Übergangserscheinungen zwischen Syntagma und komplexem Wort gibt", siehe auch Ulrich (2016: 21) sowie den Beitrag von Barz (1996), die Adjektiv-Substantiv-Syntagmen und Adjektiv-Substantiv-Kompositionen beleuchtet.

${ }^{13}$ Vgl. das Kollokationswörterbuch von Häcki Buhofer et al. (2014) für Sprachlernende sowie die Monographie von Reder zum Kollokationserwerb bei DaF-/DaZ-Lernenden.

${ }^{14}$ Vgl. aber zur Beschäftigung von Kollokationen bei Muttersprachlern die Untersuchung von Margewitsch (2005) zu „'falschen“ Kollokationen“ in schriftlichen Texten sowie die Arbeitsmaterialien von Ulrich (2016), die sich u. a. mit der Vermittlung von Kollokationen im Unterricht befasst.
}

ISSN 1615-3014 
sich zunächst im Zuge von Routinisierungsprozessen, während derer es z. B. auch zu unüblichen Verwendungsweisen kommen kann, aneignen.

Kollokationen sollten entsprechend nach Ulrich (2016: 28) - der m.W. als einziger die Arbeit mit Kollokationen auch für den muttersprachlichen Unterricht thematisiert und Materialien zur „Förderung der Sprachkompetenz durch Erwerb morphologischer Bewusstheit, Wortschatzerweiterung und Verbesserung der Ausdrucksfähigkeit" (Untertitel der Monographie) vorlegt im Unterricht ,als sprachliche Einheiten“ behandelt werden, um „die Entwicklung einer psycholinguistischen Festigkeit der jeweiligen Kollokation im mentalen Lexikon zu erreichen und dadurch deren Speicherung als Einheit zu fördern“. Ganz abgesehen davon, wie sinnvoll dies gerade auch unter linguistischen Gesichtspunkten erscheinen mag, ist für den vorliegenden Beitrag die Frage interessant, ob die SuS über diese festen Einheiten verfügen oder ob sie sie erst im Laufe ihrer schulischen Sozialisation, resp. schulisch flankiert/evoziert erwerben, was sich beispielsweise in der nicht-usuellen Kombination einer Basis mit einem Kollokator zeigen könnte.

Vor diesem Hintergrund werden aus dem Bereich der usuellen Wortverbindungen im Folgenden Funktionsverbgefüge und Kollokationen fokussiert, die sich als semantisch verbundene polylexikalische konventionalisierte Einheiten mit je einem verbalen sowie nominalen Anteil und nicht-symmetrischer Struktur auszeichnen. Im Vergleich zu einfachen Verben sind sie erstens auf stilistischer Ebene stärker konzeptionell-schriftlich geprägt und tragen zweitens in semantischer Hinsicht eine Bedeutungserweiterung/-verschiebung in sich. Sie sind vor allem in schulischen Kontexten wegen ihrer schriftsprachlichen Geprägtheit besonders relevant, stehen aber gerade deswegen den Schülerinnen und Schülern nicht bereits als ,'Fertigteile““ (Dausendschön-Gay et al. 2007: 184) in ihrem mentalen Lexikon zur Verfügung, sondern werden schulisch erworben und im Laufe der Zeit durch Routinisierungsprozesse verfestigt, d.h. ,diese Formen werden im Formulierungsprozess produziert und eben nicht reproduziert" (ebd.).

Im vorliegenden Beitrag betrachte ich ausgehend von einer Datenanalyse der vorliegenden Transkripte im Folgenden die Ausdrücke ${ }^{15}$

1. über die Ufer treten

2. Achtung erfahren

3. Generalstände einberufen,

d.h. Verbindungen des Typs Verb + Substantiv und Substantiv + Verb (vgl. Köster/Neubauer 2002: 286).

Vor dem Hintergrund der oben aufgeführten Literatur lassen sie sich wie folgt einordnen:

1. über die Ufer treten wird als Kollokation des Typs „Substantiv +Verb“ eingeordnet. Das Verb ist nicht semantisch ausgebleicht, die Kernbedeutung liegt nicht im Nominalteil. Im Kollokationswörterbuch von Häcki Buhofer et al. (2014: 852) wird der Ausdruck als Kollokation geführt.

\footnotetext{
15 Die Ausdrücke 1 und 2 entstammen nicht dem Quelltext. Es handelt es sich um die vermutete schulsprachliche (und damit konzeptionell-schriftliche) Zielform zu der von den SuS gebildeten Variante. Ausdruck 3 ist dem Quelltext entnommen. Es soll damit allerdings keinenfalls suggeriert werden, dass dies die einzige adäquate Formulierung wäre. Vielmehr sind - gerade auch mit Blick auf den Kontext der Gruppenarbeit - alternative Formulierungen denkbar.
} 
2. Achtung erfahren: Auch wenn hier sicherlich der semantische Fokus auf dem Nominalteil liegt, wird an dieser Stelle nicht dafür plädiert, von einem FVG zu sprechen, da der verbale Teil nicht semantisch ausgebleicht ist. In ihm liegt vielmehr eine Bedeutung hinsichtlich des genus verbi. So kann man jemandem entweder Achtung entgegenbringen oder in einem passivischen Sinne von jemandem Achtung erfahren. Dass dieser Bedeutungsunterschied nicht trivial ist, werden die späteren Analysen zeigen. Vor diesem Hintergrund wird Achtung erfahren hier als Kollokation des Typs „Verb + Substantiv“ gedeutet. Achtung wird auch in dem Kollokationswörterbuch von Quasthoff mit den für den vorliegenden Kontext relevanten Kollokatoren erfahren, genießen (Quasthoff 2011: 13) geführt. An diesem Beispiel zeigt sich - mit Blick auf die von den $\mathrm{SuS}$ in diesem Zusammenhang genutzte sprachliche Variante Anerkennung bekommen aber auch die Schwierigkeit, FVG und Kollokationen voneinander abzugrenzen. Im Kollokationswörterbuch von Quasthoff (2011: 23) ist „Anerkennung“ mit dem entsprechenden Kollokator „,bekommen“ als Kollokation gelistet; in dem Kollokationswörterbuch von Häcki Buhofer et al. (2014) wird Anerkennung dagegen nicht als Lemma geführt. Auch wenn ,bekommen" nicht zu den häufigsten Funktionsverben gehört, so ist es doch semantisch verblasst. Eisenberg (2006: 310) rechnet Fügungen wie „Kenntnis bekommen“, „die manchmal zu den FVG gerechnet werden“ (ebd.) zu „Konstruktionen mit <ausgebleichter> Bedeutung, die ein Nominal im Akk [...] regieren“" (ebd.).

3. Generalstände einberufen wird als Kollokation des Typs „Substantiv + Verb“ verstanden, da das Verb semantisch nicht ausgebleicht ist sowie der nominale Teil nicht die Kernbedeutung des Prädikatsausdrucks trägt. Allerdings ist Generalstände in keinem der beiden gängigen Kollokationswörterbücher als Lemma geführt. Dies lässt sich u. U. damit erklären, dass der Ausdruck spezifisch im historischen (Fach-)vokabular vorkommt, die Kollokationswörterbücher hingegen für Fremdsprachenlernende konzipiert sind und entsprechend gängige Kollokationen listen, fachsprachliche Ausdrücke hingegen ausblenden.

Wichtig dabei zu beachten ist, dass in diesem Beitrag ein weiter Kollokations-/FVG-Begriff angelegt wird, in dem der verbale Teil nicht aus nur einem möglichen Kollokator bestehen kann, sondern gewisse, Selektionsbeschränkungen unterliegende Alternativen wie in Anerkennung erhalten/Anerkennung erfahren denkbar sind.

\section{Exemplarische Analysen}

Basis der Datenauswertung ist das o.g. Korpus von video- und audiographierten problemlösenden Gruppenunterrichtsphasen sowie vorausgehenden und anschließenden Interaktionen im Klassenplenum jeweils einer niedersächsischen 5., 8. und 11. Gymnasialklasse in den Fächern Deutsch, Mathematik und Geschichte. Das Korpus ist durch Feldnotizen sowie personenbezogene Daten der Beteiligten ergänzt und in EXMARaLDA nach HIAT (Rehbein et al. 2004) transkribiert.

Für die folgenden Analysen wurde ein Teil dieses Korpus' (1 Transkript einer Gruppenarbeit in einer 5. Klasse, 3 Transkripte von Gruppenarbeiten in einer 8. Klasse jeweils im Fach $\mathrm{Ge}$ schichte) auf Kollokationen (auch nicht-usuelle Kollokationen, d. h. mit einem nicht passenden Kollokator) und FVG untersucht. Das gesamte Korpus wurde zunächst auf die genannten Strukturen hin durchsucht und mögliche entsprechende Bezugsstellen in dem medial-schriftlichen Quelltext identifiziert. Für die Analysen ausgewählt wurden schließlich ein Transkript aus einer 
fünften Klasse sowie je drei Transkripte zweier unterschiedlicher Gruppenarbeiten einer achten Klasse, da dort nach ersten Untersuchungen offenbar die meisten aktualgenetischen Erwerbsprozesse resp. die meiste Arbeit am sprachlichen Ausdruck stattfindet, während die SuS in der 11. Klasse nach bisheriger Datenlage offenbar die Gruppenarbeit nicht mehr dazu nutzen (vgl. u. a. Hee i. Dr.). Für die achten Klassen wurden jeweils drei Gruppenarbeiten zu zwei unterschiedlichen Aufgaben, allerdings unter einer von der Lehrerin vorgegebenen übergeordneten Fragestellung, untersucht. Die Zahl der drei Gruppenarbeiten ergibt sich dadurch, dass drei Schülergruppen jeweils die gleiche Aufgabe bearbeiten und so gemeinsame Formulierungspraktiken besser aufgezeigt werden können. Die Untersuchung von je drei Gruppen mit je unterschiedlichen Aufgabenstellungen und Quelltexten verfolgt zunächst ebenfalls das Ziel, gemeinsame Tendenzen in den Formulierungspraktiken aufzuzeigen, wobei die Pointe darin liegt, dass diese nun durch die unterschiedlichen Quelltexte nicht direkt und ausschließlich durch das sprachliche Material, das der Gruppenarbeit zugrunde liegt, getriggert werden und daher stärkere Aussagekraft haben. Gleichzeitig können so Auswirkungen der von der Lehrerin formulierten übergeordneten Aufgabenstellung aufgezeigt werden; in den fünften Klassen war dies nicht möglich, da die Gruppenarbeit vollständig arbeitsteilig war, d.h. jede Gruppe eine andere Aufgabe bearbeiten sollte.

Nach der Identifikation der FVG und Kollokationen im Material wurden in einem zweiten Schritt unter einer onomasiologischen Perspektive semantisch äquivalente Realisierungen resp. Wortverbindungen um den entsprechen Ausdruck in die Analysen mit einbezogen, um zu untersuchen, wie die Lerner einen semantischen Komplex sprachlich ausdrücken. Die folgenden Fallanalysen sind Ausschnitte aus Gruppenarbeitsphasen, in denen die o.g. usuellen Wortverbindungen verwendet werden resp. die Schülerinnen und Schüler an einer angemessenen Formulierung arbeiten sowie mögliche Alternativen ausprobieren. Die Analysen der sprachlichen Arbeit (vgl. Gülich/Kotschi 1995: 31) ${ }^{16}$ an diesen Wortverbindungen sowie die Verwendung möglicher ungewöhnlicher oder nicht-usueller Kombinationen sollen

a) aktualgenetische Erwerbsprozesse resp. ,intermediäre Erwerbsformate“ (Pohl 2016: 67) sichtbar machen sowie

b) Hinweise darauf geben, dass es sich bei diesen Strukturen tatsächlich nicht um feste im mentalen Lexikon als solche abrufbare sowie ad-hoc produzierbare Wortverbindungen handelt, sondern um sprachliche Strukturen, die von den Schülerinnen und Schülern erst erworben/routinisiert werden müssen.

\subsection{Fallanalyse "über die Ufer treten", 5. Klasse, Geschichte}

Kontext der Gruppenarbeitsphase, aus der die folgenden Transkriptausschnitte stammen, ist eine Geschichtsstunde in der 5. Klasse zum Thema „Lebenswelt der alten Ägypter“. Die Schülerinnen und Schüler (SuS) sind in Arbeitsgruppen aufgeteilt, die jeweils unterschiedliche Aufgaben bearbeiten. Die hier untersuchte Lernergruppe hat die Aufgabe, herauszufinden, warum der Kalender für die Ägypter wichtig war. Die SuS beziehen sich in ihren Überlegungen auf

\footnotetext{
16 Gülich/Kotschi (1995: 30f.) gehen davon aus, dass „whenever problems emerge or obstacles have to be overcome, a speaker's work of discourse production leaves traces in his/her linguistic utterance, which are then accessible to lingustic analysis.“
} 
einen Schulbuchtext, in dem die Bedeutung des Nils für das alte Ägypten thematisiert wurde. Unter anderem heißt es dort:

Während der Regenzeit im Juni strömten aus Afrikas tropischen Gebieten ungeheure Wassermassen in den Nil. In dieser Zeit stieg der Wasserstand um 5 bis 7 Meter. Ende September, wenn der Nil in sein Flussbett zurückgekehrt war, blieb zu beiden Seiten des Ufers eine Schlammschicht zurück. $[\ldots]$

Im Laufe der Zeit entdeckten die Ägypter, dass es von einer Nilüberschwemmung bis zur nächsten 365 Tage dauerte.

(Regenhardt Hrsg. 2008: 78)

Die Schülergruppe diskutiert - wahrscheinlich auf Basis dieser Informationen - darüber, dass der Kalender wichtig war, um die jährliche Überschwemmung des Nils vorauszusagen. Sie verwendet für diese Information unterschiedliche sprachliche Ausdrücke:

\begin{tabular}{l|c|}
\hline LiSuw5 [v] & $((2 \mathrm{~s}))$ Also erstmal brauchen sie damit sie \\
& \\
$7[00: 24.3]$
\end{tabular}

Die SuS greifen auf den Sachverhaltskomplex „über die Ufer treten“ zunächst mit dem alltagssprachlichen, umgangssprachlichen ${ }^{17}$ Verb überschwappen zu. Das Verb ist als Kollokator für die Basis Fluss zumindest ungewöhnlich. Im Duden Rechtschreibung (Wermke et al. Hrsg) wird es im Zusammenhang mit Essen verwendet („die Suppe ist übergeschwappt“, ebd.: 993), bei der Kollokationsdatenbank $C C D B^{18}$ lassen sich vor allem Kookkurenzen mit Emotionen (,Welle der Gewalt schwappt über, die Stimmung schwappt über, die Krise schwappt über“) finden, keinen Treffer gibt es hingegen mit einer Verbindung zu Fluss. In gängigen Kollokationswörterbüchern (Quasthoff 2011; Häcki Buhofer et al. 2014) ist das Verb nicht gelistet. Für den hier gegebenen Kontext „anschwellen“ werden vielmehr folgende Verben/Kollokationen aufgeführt: „steigt“, ,schwillt an“, „führt Hochwasser“, „tritt über die Ufer“ (Häcki Buhofer et al. 2014: 265), ,anschwellen“, ,ansteigen“, ,überschwemmen“ (Quasthoff 2011: 169).

Die SuS ersetzen in ihren folgenden Formulierungen den in diesem Zusammenhang ungewöhnlichen Kollokator, indem sie zum einen dem Kontext entsprechend passendere Kollokatoren verwenden, zum anderen ganz andere sprachliche Alternativen wählen, wie die folgenden Beispiele zeigen.

\footnotetext{
$17 \mathrm{http}: / /$ www.duden.de/rechtschreibung/ueberschwappen [22.02.2017]

$18 \mathrm{http}: / /$ corpora.ids-mannheim.de/ccdb/ [22.02.2017]
} 


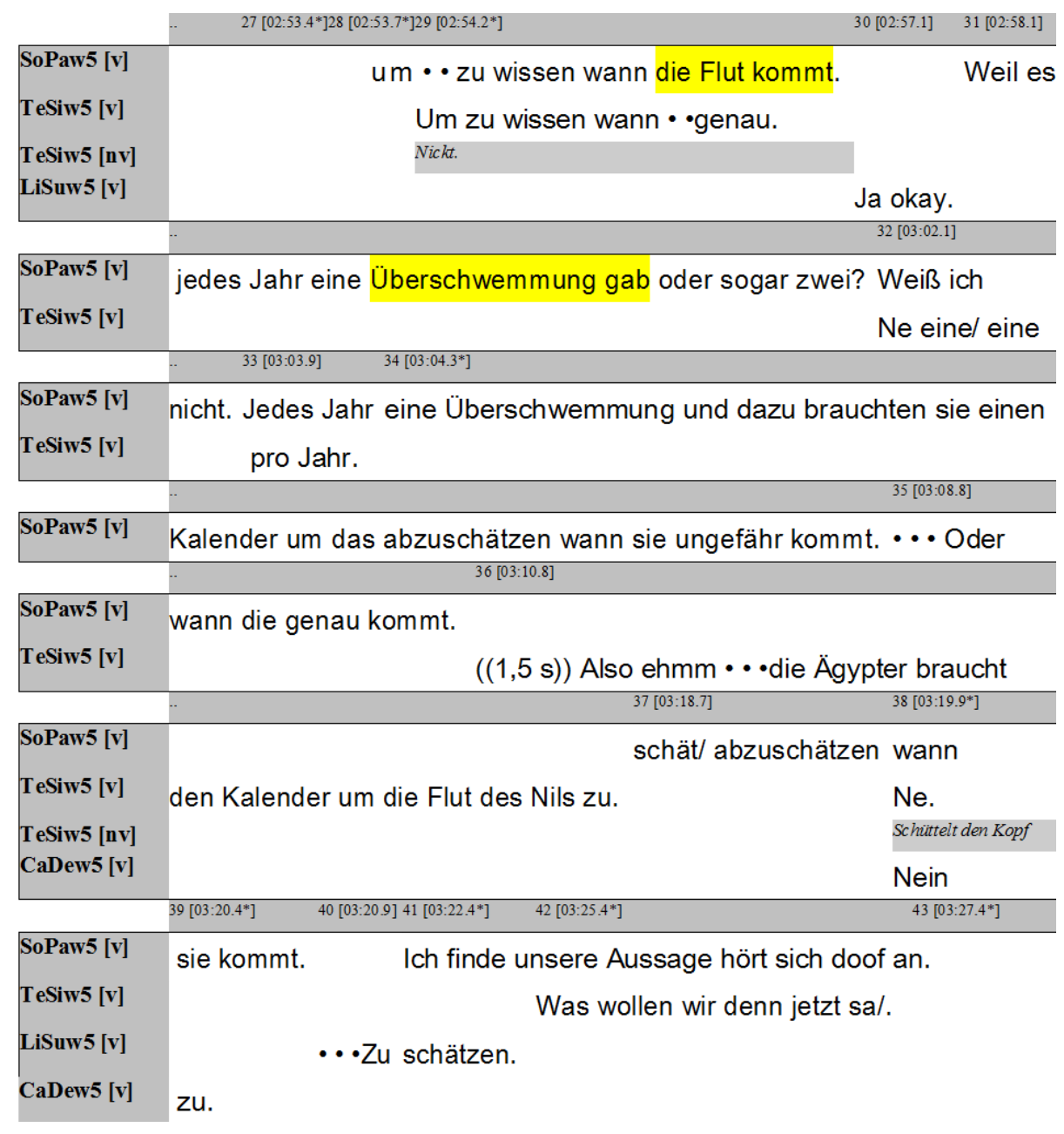

Die im weiteren zeitlichen Verlauf darauf folgende Formulierung der Lerngruppe ist eine sprachliche Alternative, die aus der Basis Flut ${ }^{19}$ und dem Kollokator kommt besteht. Diese Kombination ist eine übliche Kollokation (vgl. z. B. Quasthoff 2011: 170), wobei der Kollokator im Gegensatz zu anderen üblichen Kollokatoren wie ,einsetzen“ (ebd.) weniger fachsprachlich, aber durchaus angemessen ist.

In ihrer nächsten Verbalisierung des Sachverhaltskomplexes verwenden die SuS die im Schulbuchtext in „Nilüberschwemmung“ vorkommende Derivation Überschwemmung in Kombination mit dem Verb geben und im weiteren Verlauf in Verbindung mit dem Pronomen sie, das auf die Überschwemmung rekurriert, mit dem Verb kommen. In den beiden o.g. Kollokationswörterbüchern wird „Überschwemmung“ nicht als Lemma geführt, in der CCDB werden hauptsächlich solche Beispiele aufgeführt, in denen „Überschwemmung“ als verbalisiertes Substantiv in eine übergeordnete Phrase integriert ist, so einen integrativeren, komplexeren Satzbau ermöglicht und entsprechend nicht mit einem kookkurierenden Verb vorkommt.

Der Ausschnitt zeigt, dass die SuS um die richtige Formulierung ringen, sie ergänzen sich dabei gegenseitig (Turn 36-39: TeStw5: „Also ehmm • • die Ägypter braucht den Kalender um die Flut des Nils zu“ - SoPaw5: „schätz/abzuschätzen wann sie kommt“). Alle Gruppenmitglieder sind mit ihrer Formulierung nicht zufrieden. So evaluiert TeStw5 ihre Äußerung mit einem

\footnotetext{
${ }^{19}$ Flut wird hier nicht im Sinne der Gezeiten, sondern als „größere, strömende Wassermasse“ (http://www.duden.de/rechtschreibung/Flut, [28.02.2017]) verstanden. In dieser Bedeutung wird es laut Duden einem „gehoben[em]" Register zugeordnet (ebd.).
} 
kopfschüttelnden „ne“, CaDew5 mit „Nein“ und SoPaw5 macht es metasprachlich explizit: „Ich finde unsere Aussage hört sich doof an“ (Partiturfeld 41-42). Die SuS scheinen sich darüber im Klaren zu sein, dass bei der im Anschluss an die Gruppenarbeitsphase angekündigten Ergebnispräsentation im Klassenplenum von ihnen eine spezifische Art von Äußerung erwartet wird. Dieser Erwartung, die wahrscheinlich mit schultypischen Sprachstrukturen und Mustern in Verbindung zu bringen ist, scheinen sie gerecht werden zu wollen. Sie unternehmen weitere Formulierungsversuche:

(3)

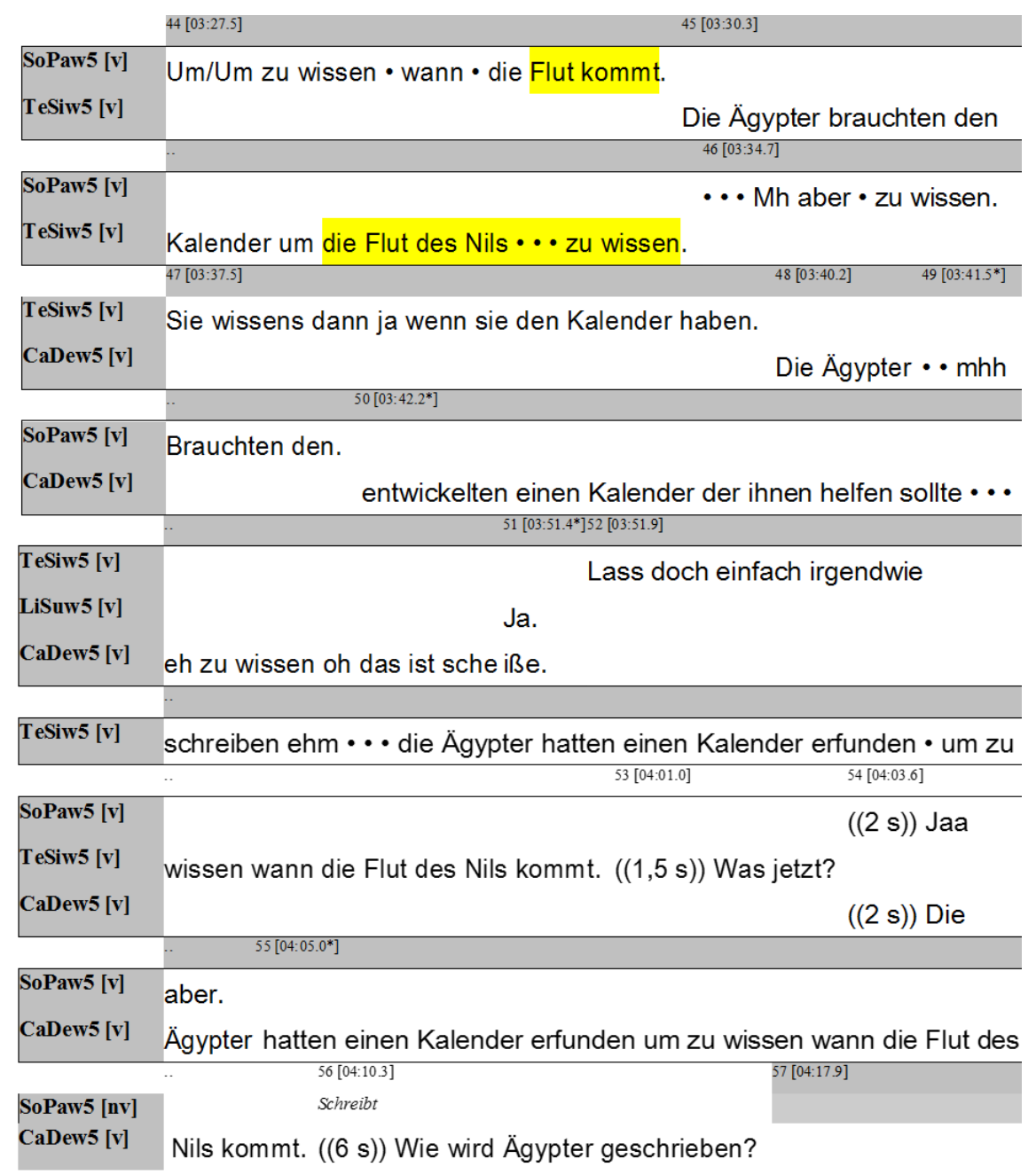

SoPaw5 greift zunächst auf die bereits vorher von ihr formulierte Wendung um zu wissen, wann die Flut kommt zurück, was von TeSiw5 zum einen in einen übergeordneten Satz integriert wird: Die Ägypter brauchten den Kalender, um die Flut des Nils $・ \cdots$ zu wissen; zugleich unternimmt sie dabei den Versuch, den von ihrer Mitschülerin formulierten finalen Nebensatz stärker zu integrieren, indem sie versucht, den temporalen Nebensatz in die Nominalphrase zu integrieren. Dies scheitert, da nun eine unangemessene Kollokation ,, die Flut wissen“ entsteht. Diese entsteht möglicherweise durch die grammatische Inkongruenz, d. h. der Realisierung eines sprachlichen Elements durch eine inkongruente Wortart (wie es z. B. bei Nominalisierungen der Fall ist) (vgl. Pohl 2007: 420): Die SuS integrieren den temporalen Nebensatz in eine Nominalphrase, wobei der Prozess der grammatischen inkongruenten Realisierung nicht glückt, da das temporale Element wann die Flut kommt nicht in eine inkongruente Realisierung (wie z. B. ,den Zeitpunkt der Überflutung“) überführt, sondern weggelassen wird. Die SuS sind mit 
dieser Formulierung nicht zufrieden und starten einen neuen Versuch, bei dem die gescheiterte integrative Struktur in einer Nebensatzkonstruktion aufgelöst wird: entwickelten einen Kalender, der ihnen helfen sollte $\cdots$ - eh zu wissen. Die Schülerin bricht die Konstruktion, die im Vergleich zu der vorherigen Formulierung nun mit einer Nebensatz-Konstruktion 3. Grades weitergeführt werden müsste, ab. Sie ist mit der Formulierung nicht zufrieden: oh das ist scheiße. Im nächsten Anlauf tilgen die SuS den Relativsatz und damit eine Nebensatz-Ebene und drücken den Sachverhalt nun in einer nicht-integrierten, allerdings im Vergleich zur vorherigen Formulierung etwas weniger komplexen Nebensatzkonstruktion aus: hatten einen Kalender erfunden um zu wissen wann die Flut des Nils kommt. Im Anschluss finden die SuS eine weitere Formulierungsalternative:

\begin{tabular}{|l|l|}
\hline CaDew5 [v] & $((2 \mathrm{~s}))$ Hatten $((2 \mathrm{~s}))$ einen $((4 \mathrm{~s}))$ Kalender $((10 \mathrm{~s}))$ um zu \\
\hline CaDew5 [v] & wissen/ $/($ [05:11.0] $\mathrm{s}))$ um zu wissen wann der Nil über die Ufer ging. \\
\hline
\end{tabular}

Die SuS verwenden hier die bisher noch nicht verwendete Alternative über die Ufer gehen, die im Folgenden um über die Ufer treten ergänzt wird:

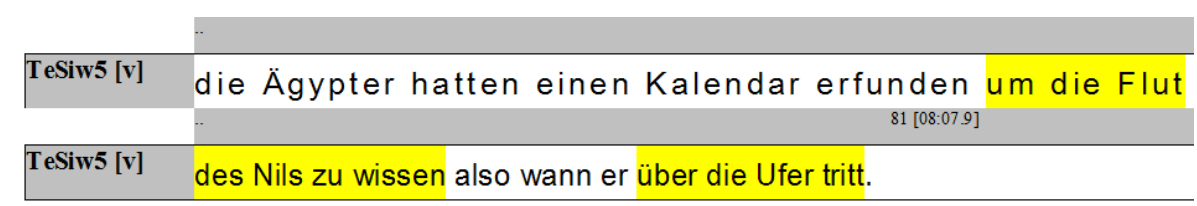

Im „Wörterbuch der Kollokationen im Deutschen“ (Quasthoff 2011: 464) findet sich keine Entsprechung für den semantischen Komplex „Überschwemmung“, also bspw. ,über die Ufer gehen“ oder „über die Ufer treten“. Im Kollokationswörterbuch von Häcki Buhofer et al. (2014: 852) wird unter dem Lemma „Ufer“ als Beispiel unter dem Verb „überschwemmen“ dagegen die Kollokation ,,über die Ufer treten“ aufgeführt: „ein Fluss/ein Bach/... steigt ${ }^{\circ}$ | tritt über die U.“ (ebd.). Auch in der CCDB lassen sich viele Häufigkeiten für die Kookurrenz von „Ufer“ und „treten“ finden, dagegen gibt es keine für „Ufer“ und „gehen“.

Die hier beobachteten SuS nutzen in der Gruppenarbeit insgesamt folgende Formulierungsalternativen: überschwappen, Überschwemmung geben, Flut kommen, über die Ufer gehen, über die Ufer treten.

Bezieht man andere Formulierungen von SuS aus anderen Gruppen mit ein, zeigt sich, dass einige Formulierungen identisch sind (,,über die Ufer treten“), andere das Formulierungs-Spektrum erweitern: „es kommt zu großen Überschwemmungen“ (statt wie in der hier fokussierten Gruppe „Überschwemmungen geben“) sowie die integrative Konstruktion „,bei großen Überschwemmungen war das Feld kaputt", bei der durch die integrative Struktur der Nominalisierung das verbale Element, der Kollokator, nicht realisiert ist.

\subsection{Fallanalyse "Achtung erfahren", 8.Klasse, Geschichte}

Das zweite Fallbeispiel entstammt einer Unterrichtseinheit im Geschichtsunterricht zur Französischen Revolution resp. zur Krise in Frankreich im späten 18. Jahrhundert. Unterthema der Geschichtsdoppelstunde sind die sog. cahier de doléances (Beschwerdehefte), die die Stände 
an die Abgeordneten der Generalstände und zur Weiterreichung an den König verfasst hatten. Die Gruppenarbeit ist arbeitsteilig organisiert, d. h., es gibt nicht eine Aufgabe für den gesamten Klassenverband, sondern es werden unterschiedliche Aufgaben bearbeitet, wobei meist drei Gruppen parallel zur selben Frage arbeiten. Die hier untersuchten drei Arbeitsgruppen beschäftigen sich alle mit dem cahier de doléance des femmes (vgl. Anhang), in dem Frauen um mehr Rechte, u. a. eine Schulbildung, bitten. Der von der Lehrerin an der Tafel festgehaltene Arbeitsauftrag besteht darin, zunächst die Quelle vorzustellen sowie anschließend in eigenen Worten die Probleme vorzutragen, die in der Quelle angesprochen werden.

Die Fallanalysen beziehen sich auf mehrere Schülergruppen, die jeweils die gleiche Textstelle im Schulbuch bearbeiten:

Wir bitten um Ausbildung und Beruf, nicht, um uns die Befugnisse der Männer anzueignen, sondern um von Ihnen höher geachtet zu werden.

(Regenhardt Hrsg. 2008: 49)

Die SuS nehmen auf diese Textstelle wie folgt Bezug:

(6)

\begin{tabular}{|c|c|}
\hline & $219[21: 05.2]$ \\
\hline HaErm8 [v] & ((7 s)) Damit sie ((unverständlich))/ \\
\hline \multirow[t]{2}{*}{ HaZlm8 [v] } & ((20 s))Bitten um Ausbildungschancen. \\
\hline & $221[21: 39.2]$ \\
\hline HaErm8 [v] & damit sie von den Männern mehr anerkannt werden. \\
\hline \multirow[t]{2}{*}{ FiLim8 [v] } & -. Ehh, mehr Anerkennung und. \\
\hline & $.222[21: 42.5]$ \\
\hline HaErm8 [v] & Um mehr Anerkennung zu ve/ um mehr Anerkennung von den Männern zu \\
\hline FiLim8 [v] & Ja genau. \\
\hline & $225[21: 56.2]$ \\
\hline
\end{tabular}

HaErm8 nimmt auf die Textstelle Bezug, indem er die syntaktische Struktur beibehält und höher und geachtet durch die bedeutungsähnlichen Ausdrücke mehr und anerkannt ersetzt. Die passivische Semantik bleibt dabei erhalten. Im Anschluss unternimmt FiLim8 unter einigem Planungsaufwand (vgl. die Pause ••• sowie die gefüllte Pause $e h$ ) den Versuch einer Nominalisierung. Dieser scheitert allerdings an der Phrasenexpansion resp. der syntaktischen Integration, da der entsprechende Kollokator nicht von FiLim8 realisiert wird. Das passivische Moment, das durch die Nominalisierung eigentlich in einen verbalen Kern ausgelagert werden müsste, geht dadurch verloren. HaErm8 übernimmt in seinem Folgeturn die Idee, den Sachverhalt nominalisiert auszudrücken. Auch hier zeigt sich der Planungsaufwand in Form eines Abbruchs und einer durch eine Planungspause eingeleiteten Wiederholung (mehr Anerkennung), die phrasal und syntaktisch durch von den Männern zu bekommen erweitert ist. In dieser Formulierung ist die passivische Aspektualisierung durch die Präpositionalphrase von den Männern sowie der Kollokator mit passivischer Bedeutung bekommen realisiert. Phrasenexpansion und syntaktische Integration gelingen in Kombination mit der Wahl einer eher neutralen Kollokation resp. mit einem Passepartout-Verb als Kollokator (Anerkennung bekommen). Der Kollokator ist im Wörterbuch von Quasthoff (2011: 23) neben eher konzeptionell-schriftlichen Kollokatoren wie „erfahren“, „erhalten“ „erlangen“ als Möglichkeit geführt. Auffällig ist an diesem Ausschnitt, dass die SuS bei Nominalisierungen entweder keine (wie bei FiLim8) oder tendenziell eher 
Kollokatoren verwenden, die als Passepartout-Verben (bekommen) eher zu den nähesprachlichen Struktur- und Ausdrucksformen gerechnet werden können (vgl. Koch/Oesterreicher 1986: 27). Schriftsprachlich-affizierte Kollokatoren (wie die bei Quasthoff genannten) werden hingegen nicht verwendet. Diese Tendenz zeigt sich auch in der Präsentation:

(7) Präsentation:

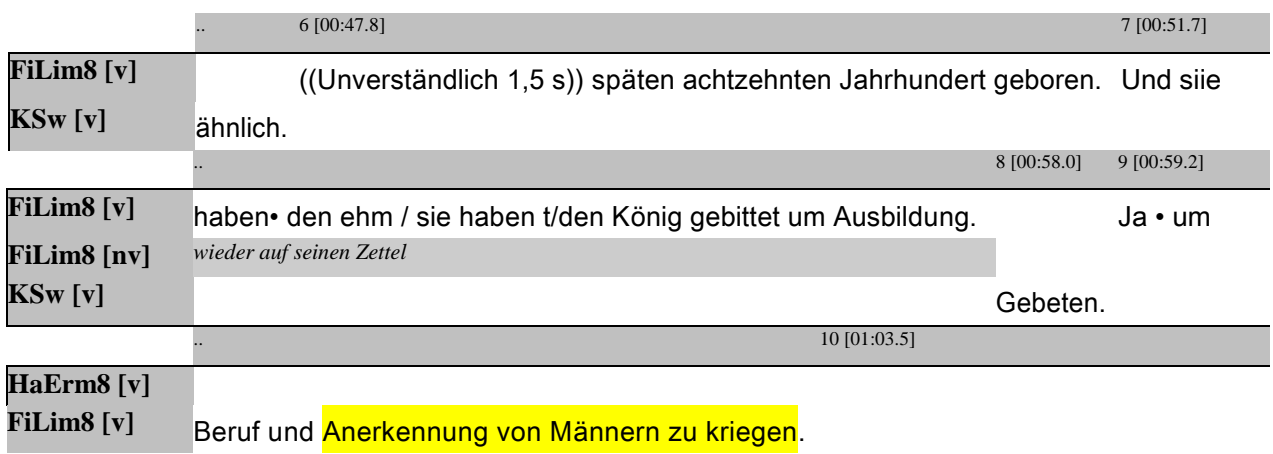

FiLim8 übernimmt wörtlich die in der Gruppenarbeitsphase erarbeitete Formulierung, ersetzt allerdings $z u$ bekommen durch das umgangssprachliche Verb zu kriegen. ${ }^{20}$ Man sieht also auch hier, dass es den Lernenden offensichtlich Schwierigkeiten bereitet, eine dem schulischen Kontext angemessene, konzeptionell-schriftliche Kollokation zu finden (bspw. „erfahren“, „erhalten“, „finden“" oder „genießen"). ${ }^{21}$

Auch in einer weiteren Gruppe, die sich mit dem gleichen Text beschäftigt, wird der im Text verwendete Ausdruckt ,geachtet werden“ nominalisiert und auch hier zeigen sich Probleme beim Finden des richtigen Kollokators:

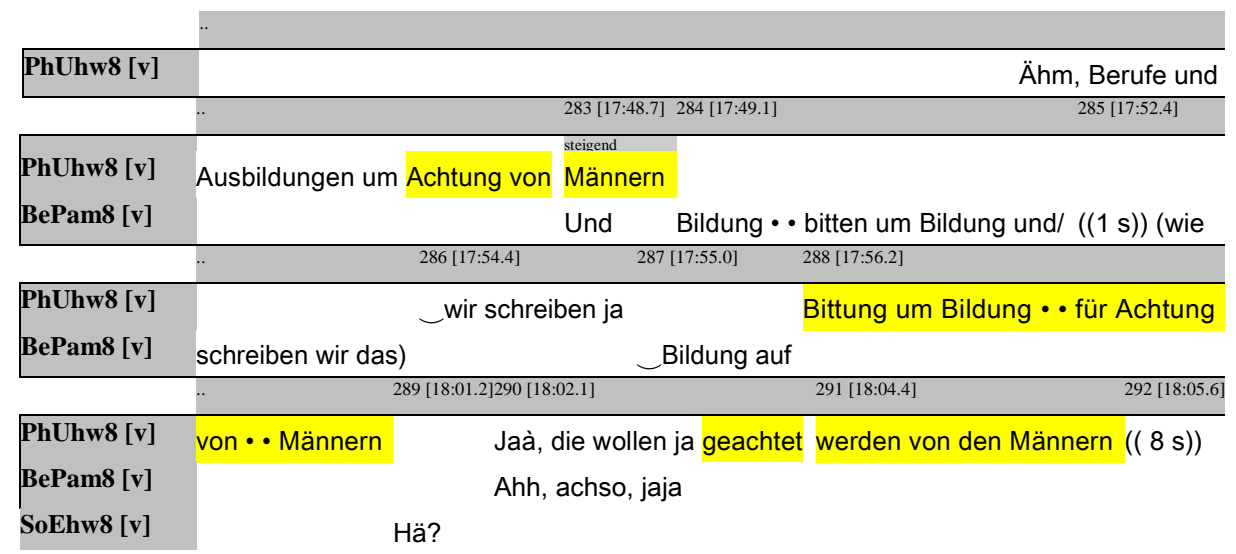

Der Text wird zunächst vorgelesen und direkt im Anschluss von PhUhw8 paraphrasiert, indem sie mit Achtung von Männern eine Nominalisierung bildet. Die Gruppe entscheidet sich im Vergleich zur vorherigen Gruppe, die Anerkennung als Basis gewählt hatte, für die Basis Achtung, die als grammatische Metapher eine größere Nähe zum Ausgangstext aufweist.

\footnotetext{
${ }^{20}$ Der Duden online listet „zu bekommen“ unmarkiert als „Umschreibung des Passivs“, während „kriegen“ als umgangssprachliches Synonym markiert wird.

${ }^{21}$ Die Kollokationen „Anerkennung finden“ bzw. „Anerkennung genießen“ sind als Synonyme von anerkannt werden in der von Helbig \& Buscha (1993: 84 u. 87) zusammengestellten Liste der Funktionsverbgefüge gelistet, „erfahren“, „erhalten“ als mögliche Kollokatoren bei Quasthoff (2011: 23).
} 
Auch in dieser Gruppe wird die Nominalisierung nicht mit einem Kollokator realisiert und nicht syntaktisch integriert und verliert so das passivische Moment, was durch die Präposition vor statt ,von" noch verstärkt wird (aktivische Bedeutungsverschiebung). Die beobachtete Tendenz zur Nominalisierung zeigt sich auch in der nächsten Formulierung von PhUhw8: Bittung um Bildung •・• für Achtung von • Männern. Die Schülerin bildet hier mehrere Nominalisierungen durch -ung-Derivationen, was zu einer Übergeneralisierung in Bittung führt. Die Konstruktion ist darüber hinaus so komplex, dass sie von der Mitschülerin SoEhw8 nicht verstanden wird. Interessanterweise löst PhUhw8 sie nun im Folgeturn verständnissichernd als Verbalisierung, also in eine syntaktisch weniger integrative Form, auf. Diese Übergeneralisierung sowie die viergliedrige komplexe Nominalphrase scheinen für die Gruppe offensichtlich eine besonders gelungene Formulierung zu sein: Sie wird wortwörtlich so aufgeschrieben und im späteren Verlauf der Gruppenarbeit als Musterlösung wiederholt. Durch die Nominalisierung und damit einhergehende grammatische Inkongruenz (sensu Halliday 1985; 1988 , vgl. Pohl 2007: 417), d.h. der Überführung von einer Wortart in eine andere, muss der entsprechende Kollokator für Achtung nicht realisiert werden, sondern wird durch eine Präpositionalphrasenstruktur Höherachtung bei Männern ausgedrückt:

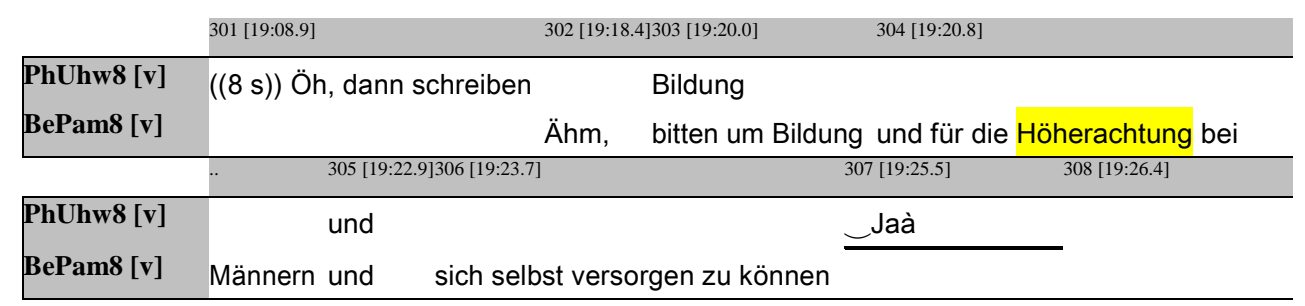

Eine weitere grammatische Inkongruenz (sensu Halliday 1985; 1988 , vgl. Pohl 2007: 417), zeigt sich in dem Kompositum Höherachtung, das ursprünglich aus dem im Text vorkommenden adverbialen „höher“ und dem Verb in passivischer Konstruktion ,geachtet werden“ gebildet wird.

In der Präsentation greifen die SuS auf die in der Gruppenarbeit als Musterlösung aufgeschriebene viergliedrige Nominalphrase zurück, die allerdings verständnissichernd in einem finalen Nebensatz aufgelöst wird, wodurch auch die Übergeneralisierung Bittung wegfällt. Bestehen bleibt die Herausforderung, eine für den schulischen Kontext angemessene, ,bildungssprachliche' Kollokation zu bilden. Stattdessen wählen sie eine zulässige, allerdings eher neutrale, weniger elaborierte Variante mit einem Passepartout-Verb:

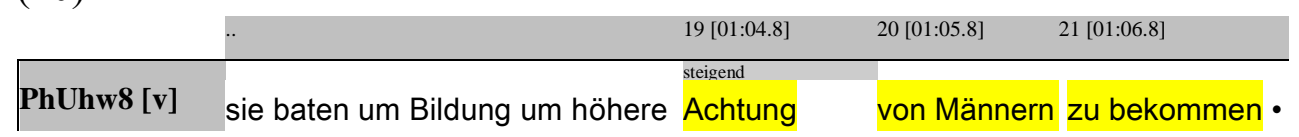

Werfen wir abschließend einen Blick auf eine dritte Gruppe: 


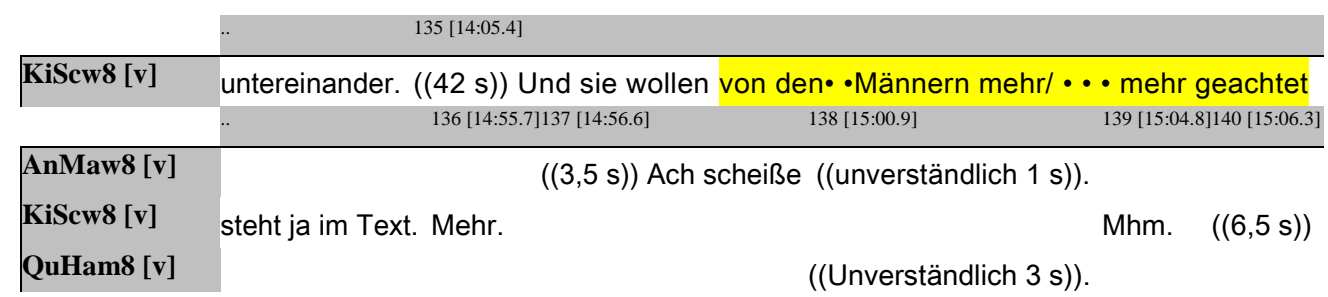

Die Gruppe greift zunächst auf die im Text verwendete Formulierung zurück. Dies scheint allerdings nicht akzeptabel (steht ja im Text), was dazu führt, dass sie nicht weiter verwendet wird und die SuS offensichtlich in Formulierungsnot bringt, worauf die langen Pausen, das metakognitive Ach scheiße sowie das Unsicherheit und Nicht-Weiter-Wissen-ausdrückende Mhm schließen lassen. Die SuS suchen im Folgenden nicht nach einer alternativen Formulierung, sondern widmen sich einem anderen Themenkomplex. Im späteren Verlauf der Gruppenarbeit kommen sie noch einmal auf den Sachverhalt zurück:

\begin{tabular}{|l|l|}
\hline AnMaw8 [v] & haben. $\cdots$ Und ehmm $((2 \mathrm{~s}))$ mehr Achtung der Männer, $\cdots$ dass sie halt nicht so $\cdots$ \\
\hline & \\
\hline 236 [22:19.5] $\cdots 237[22: 20.1]$ \\
\hline AnMaw8 [v] & von den Männern $\bullet(($ unverständlich $))$.
\end{tabular}

In der Gruppe werden abgesehen von dieser Formulierung keine weiteren Formulierungsalternativen erarbeitet. AnMaw8 paraphrasiert den Text, indem sie, wie die SuS der anderen Gruppen auch, eine Nominalisierung bildet. Auch sie realisiert keinen Kollokator, was zu inhaltlichen Problemen führt: Durch die verwendete ambige Struktur mit „der“ sowie das fehlende Prädikat geht die passivische Semantik verloren. Dies könnte eine Erklärung dafür sein, warum QuHam8 die Sachlage in der Präsentation inhaltlich falsch darstellt:

\begin{tabular}{llrr} 
& \multicolumn{1}{l}{} & & $16[01: 02.4]$ \\
\hline QuHam8 [v] & & $17[01: 04.8]$ & Also sie \\
\hline QuHam8 [v] & haben ke/keine Achtung vor den Männern. &
\end{tabular}

Das von dem Schüler genutzte FVG Achtung haben, bei dem das Verb semantisch ausgebleicht ist und damit die passivische Bedeutung verloren geht, bekommt im Zusammenhang mit der Präposition vor eine neue, aktivische Bedeutung, denn die Frauen wollen „,von den Männern geachtet werden“ und haben nicht etwa ,keine Achtung vor den Männern“.

Das Beispiel zeigt, dass die Arbeit an grammatischen Inkongruenzen (sensu Halliday 1985; 1988 , vgl. Pohl 2007: 417) und das Bilden der richtigen Kollokation für die SuS nicht trivial ist. In dieser Gruppe stellt sich dies gar als Problem heraus, das sich auf den Inhalt auswirkt: Die Anforderung, eine angemessene Kollokation mit Achtung und passivischer Semantik zu wählen wie (z.B. „erfahren“, Quasthoff 2011: 13, ,erwerben“ Häcki Buhufer et al. 2014: 7), mündet in eine inhaltlich falsche Darstellung.

\subsection{Fallanalyse "Generalstände einberufen", 8. Klasse, Geschichte}

Das dritte Fallbeispiel entstammt dem gleichen Geschichtsunterricht, der bereits im zweiten Fallbeispiel Grundlage der Daten war. Im Gegensatz zu den oben behandelten Gruppen beschäftigen sich die hier fokussierten Gruppen mit dem cahier de doléance der Bauern (vgl. 
Anhang). Der von der Lehrerin an der Tafel festgehaltene Arbeitsauftrag besteht wie bei der anderen Gruppe auch darin, zunächst die Quelle vorzustellen sowie anschließend in eigenen Worten die Probleme vorzutragen, die in der Quelle angesprochen werden. Im Folgenden geht es um diesen Textausschnitt:

Der König wird demütig gebeten, die Generalstände alle fünf Jahre einzuberufen, um von unserem Unglück zu erfahren.

(Regenhart Hrsg. (2009: 49)

Der Text wird von insgesamt drei Schülergruppen bearbeitet. Sie alle gehen unterschiedlich mit dem fachsprachlichen ${ }^{22}$ Ausdruck „Generalstände einberufen“ um. Die beiden o.g. Kollokationswörterbücher listen „Generalstände“ nicht als Lemma, was sich mit der Fachsprachlichkeit des Ausdrucks erklären lassen könnte. Bei der CCDB lassen sich zu „Generalstände“ die verbalen Kookkurrenzen ,einberufen“ (14), ,zusammentreten“(4), ,wählen“ (3) sowie ,ausrufen“ (1) finden, wobei „einberufen“ die größte Häufigkeit zeigt, vor allem, wenn man die Nominalisierung „Einberufung“ (28) mit einbezieht (mit Verbal- und Nominalform insg. 42 Kookkurrenzen).

\begin{tabular}{|c|c|}
\hline $\begin{array}{l}\text { CeSaw8 [v] } \\
\text { NiKlw8 [k] }\end{array}$ & $\begin{array}{l}((35 \mathrm{~s})) \\
\text { Gespräch über das Aufnahmegerät }\end{array}$ \\
\hline \multirow[t]{2}{*}{ CeSaw8 [v] } & ruft der König alle fünf Jahre die Generalstände zusammen, um ((2 s)) vom ((3 s)) \\
\hline & $288[34: 34.4]$ \\
\hline CeSaw8 [v] & keine Ahnung. \\
\hline CaDiw8 [v] & $((1,5 \mathrm{~s}))$ ((Unverständlich)). \\
\hline
\end{tabular}

Der Erstzugriff der SuS erfolgt über einen Kollokator zusammenrufen, der vom Sprachmaterial an den fachsprachlichen Kollokator einberufen angelehnt ist, gleichzeitig aber eher umgangssprachlich affiziert ist. Die Äußerung wird hier von der Schülerin nicht weitergeführt, weil sie den Satz (wahrscheinlich inhaltlich) nicht fortsetzen kann. Der Abbruch scheint dagegen nichts mit der unangemessenen Verwendung der Kollokation zu tun zu haben; es ist offensichtlich kein metakognitives Bewusstsein darüber aktiviert, dass die Kollokation so nicht gebräuchlich ist. Etwas später im Text kommen die SuS noch einmal auf den Sachverhalt zurück. Die gleiche Schülerin wie im Transkriptausschnitt (14) versucht sich an einer alternativen Formulierung:

\footnotetext{
22 Es wird in diesem Zusammenhang davon ausgegangen, dass Generalstände einberufen ein lediglich für historische Kontexte resp. in historischen Kontexten gebräuchlicher Ausdruck und damit fachsprachlich (affiziert) ist.
} 


\begin{tabular}{|c|c|}
\hline & $295[35: 03.9]$ \\
\hline CeSaw8 [v] & • - Der König soll alle fünf Jahre, die \\
\hline CaDiw8 [v] & ((Unverständlich)). \\
\hline \multirow[t]{2}{*}{ LeRaw8 [v] } & ((Unverständlich)). Alle fünf Jahre • äääh. \\
\hline & $297[35: 10.8] \quad 298[35: 11.8]$ \\
\hline CeSaw8 [v] & ((Unverständlich)). ••Also ••der König. \\
\hline CaDiw8 [v] & $((9 \mathrm{~s}))$ \\
\hline \multirow[t]{2}{*}{ LeRaw8 [v] } & Einberufen. \\
\hline & $301[35: 41.2]$ \\
\hline CeSaw8 [v] & ((32 s))Was sind Generalstände? Das sind doch diie ehm höheren \\
\hline \multirow[t]{2}{*}{ CaDiw8 [v] } & Was schreibt ihr? \\
\hline & 303 [36:16.9] \\
\hline \multirow[t]{2}{*}{ CeSaw8 [v] } & Stände, oder? ((5 s)) Sie hat ja gesagt, wir sollen ja möglichst in eigenen Worten. \\
\hline & 304 [36:24.1] \\
\hline \multirow[t]{2}{*}{ CeSaw8 [v] } & ((Unverständlich)) wenn wir generell/ Generalstände schreiben ((unverständlich)). \\
\hline & $305[36: 28.1] \quad 306[36: 31.8]$ \\
\hline CeSaw8 [v] & $((1,5 \mathrm{~s}))$ (Generalstände) wollen wir hinschreiben. \\
\hline \multirow[t]{2}{*}{ CaDiw8 [v] } & • Ich weiß ja nich, was das heißt. \\
\hline & $308[36: 36.4] \quad 309\left[36: 38.8^{*}\right] \quad 310[36: 39.5 *]$ \\
\hline CeSaw8 [v] & - Kann jemand von euch fragen? \\
\hline CaDiw8 [nv] & Meldet sich \\
\hline
\end{tabular}

Es zeigt sich, dass die Schülerin erneut Probleme mit dem Kollokator hat, was sich an der gefüllten Pause und dem Abbruch zeigt. Eine Mitschülerin springt ein und ergänzt um den fachsprachlichen Kollokator einberufen. Gleich darauf zeigt sich, dass CeSaw8, die den Ausdruck ja bereits in (14) und auch in (15) verwendet hat, keine inhaltliche Vorstellung davon hat, was Generalstände überhaupt sind (Partiturfeld 301ff.). Sie hat in ihrer Darstellung also bisher einen Ausschnitt des Originaltextes paraphrasiert, ohne ihn verstanden zu haben. Es zeigt sich in diesem Transkriptteil noch ein weiteres Problem: Die Aufgabenstellung der Lehrerin war, den Text in eigenen Worten zusammenzufassen. Nun stehen die SuS vor dem Problem, den Ausdruck Generalstände einberufen, der ja im Text so wortwörtlich verwendet wird, mit eigenen Worten ausdrücken zu müssen. Dadurch ergibt sich ein doppeltes Problem. Das erste zeigt sich aus Schülerperspektive: Die SuS haben den Ausdruck inhaltlich nicht verstanden und können ihn deshalb nicht paraphrasieren. Das zweite Problem ergibt sich aus einer sprachlich-fachlichen Perspektive und ist dem Problem aus Schülerperspektive quasi entgegengesetzt: Die Aufgabenstellung verleitet die SuS dazu, nach sprachlichen Alternativen zu suchen, wo es keine gibt, denn es handelt sich um einen festen Ausdruck, der nicht mit eigenen Worten widergegeben werden kann, ohne dass dadurch ein semantischer Verlust mit einhergehen würde. Die SuS holen sich im Anschluss Hilfe bei der Lehrerin, die ihnen den Ausdruck Generalstände erklärt. Direkt an die Erläuterungen der Lehrkraft anschließend greift CeSaw8 ihre ursprüngliche Formulierung, die sie um einen erklärenden Relativsatz ergänzt, wieder auf: 
(16)

\begin{tabular}{|c|c|}
\hline & $325[38: 38.5] 326[38: 39.8]$ \\
\hline \multirow[t]{2}{*}{ CeSaw8 [v] } & •. Also ich hab jetzt geschrieben: Der König soll alle fünf Jahre die \\
\hline & $327[38: 48.0]$ \\
\hline \multirow[t]{2}{*}{ CeSaw8 [v] } & Generalstände, das sind die obersten aller drei Stände • zusammenberufen. $((4,5 \mathrm{~s}))$ \\
\hline & $328[38: 53.5] 329[38: 55.5]$ \\
\hline CeSaw8 [v] & Zusammenberufen? \\
\hline CaDiw8 [v] & •. Jaa. \\
\hline
\end{tabular}

Sie ist sich offensichtlich bezüglich des Kollokators unsicher, weshalb sie nachfragt (zusammenberufen?), wird aber von ihrer Mitschülerin CaDiw8 positiv bestärkt. Es folgt keine weitere Auseinandersetzung über die richtige Formulierung. In der weiteren Gruppenarbeit wird die Kollokation in dieser Form noch ein weiteres Mal verwendet, in der anschließenden Präsentation verwendet CaDiw8 sie ebenfalls mit dem unpassenden Kollokator zusammenberufen. Sie werden von der Lehrerin nicht korrigiert, die Verwendung der unangemessenen Kollokation bleibt den SuS entsprechend unklar. Sie haben den Fachausdruck nicht gelernt. Auch bei der zweiten beobachteten Gruppe zeigen sich Probleme im Umgang mit der fachsprachlichen Kollokation:

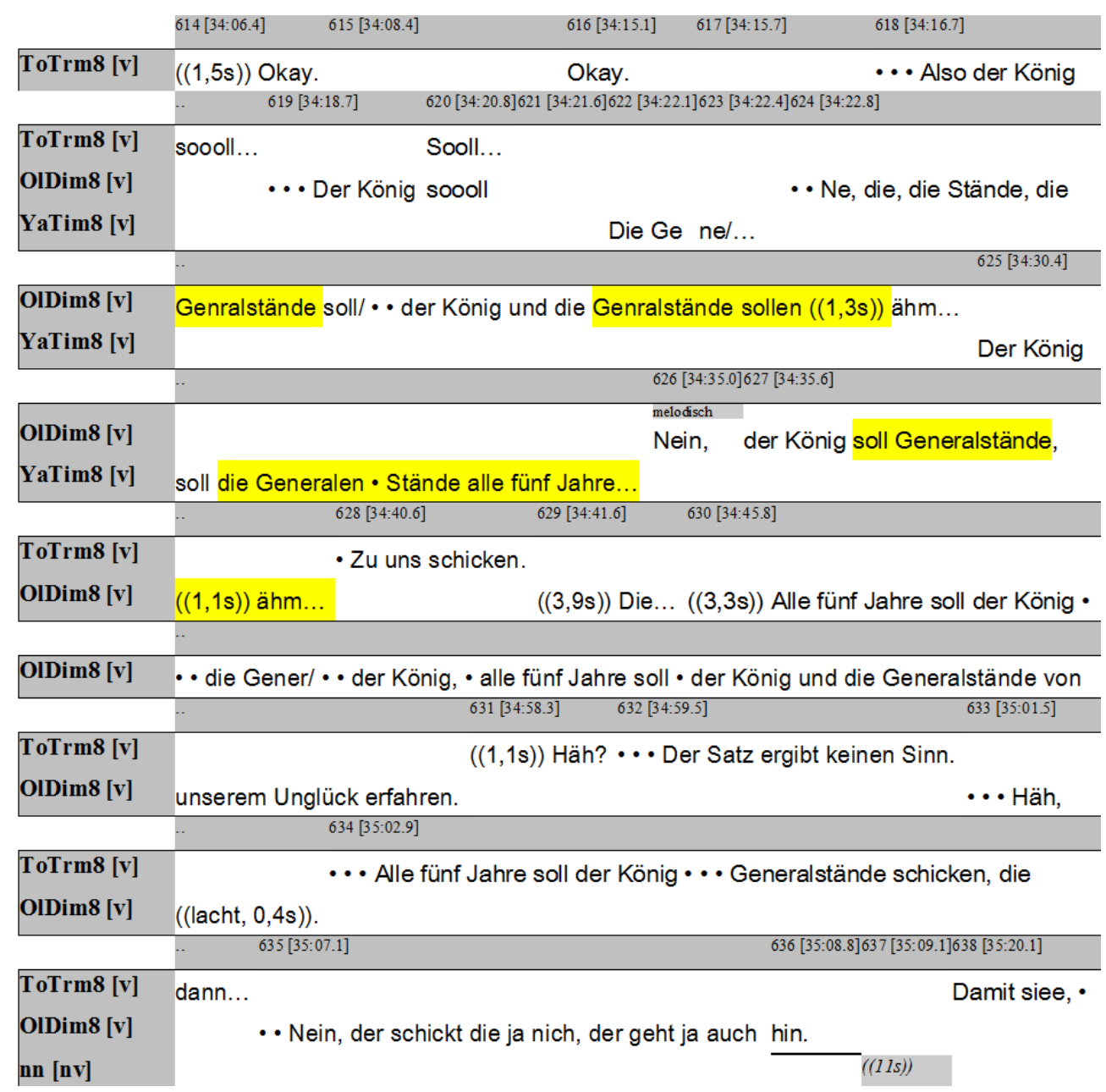




\begin{tabular}{|c|c|}
\hline & $639[35: 25.6]$ \\
\hline \multirow{3}{*}{ OIDim8 [v] } & - von unseren e/ $\cdots$ Problemen $((1,1 \mathrm{~s}))$ uund äh... \\
\hline & $\cdots$ Alter, $\cdots$ alle fünf Jahre \\
\hline & $641[35: 29.2]$ \\
\hline \multirow[t]{2}{*}{ OIDim8 [v] } & $((1,7 \mathrm{~s}))$ ähm, $\cdots$ soll der König die Generalstände einberufen, $\cdots$ also \\
\hline & $642[35: 37.4]$ \\
\hline \multirow[t]{2}{*}{ OIDim8 [v] } & ((unverständlich, $0,3 \mathrm{~s}))$ wir jetz wie ((unverständlich, 0,2s)) abgeschrieben ${ }^{\circ}((2,1 \mathrm{~s})) \mathrm{Ah}$, \\
\hline & $643[35: 40.4] \quad 644[35: 45.7]$ \\
\hline ToTrm8 [v] & $((4,3 \mathrm{~s}))((\text { unverständlich, } 0,9 \mathrm{~s}))^{\circ}$ \\
\hline OIDim8 [v] & $((4,3 s))$ Ich frag sie mal einfach, \\
\hline
\end{tabular}

Die vielen Abbrüche und gefüllten wie ungefüllten Pausen zeigen, dass die SuS Schwierigkeiten haben, die richtige Formulierung zu finden. Auch die weitere Auseinandersetzung zeigt, dass die SuS offensichtlich sowohl inhaltliche als auch sprachliche Schwierigkeiten haben: Nein, der schickt die ja nich, der geht ja auch hin. OlDim8 macht im Anschluss einen Formulierungsvorschlag: soll der König die Generalstände einberufen. Dieser ist ebenfalls durch lange Pausen gerahmt und wird anschließend verständlicherweise von dem Schüler selbst wieder verworfen, weil der Ausdruck so auch im Text vorkommt, der Arbeitsauftrag aber ausdrücklich war, eigene Worte zu verwenden. Unsinnigerweise wird der angemessene Ausdruck also auf Grund der Aufgabenstellung aufgegeben. Die SuS diskutieren im Anschluss, ob sie Hilfe bei der Lehrerin (KSw) holen sollen, entscheiden sich zunächst aber dagegen, da sie den Satz verstehen und nur nicht wissen, wie sie es formulieren sollen. Nach mehreren weiteren Anläufen, in denen sie keine Formulierungsalternativen finden, dabei den Satz immer wieder verblos abbrechen, rufen sie nun doch die Lehrerin um Hilfe: „• • Wir wissen nich wie wir den schreiben sollen in eigenen Worten“. Die Lehrerin greift im Folgenden allerdings lediglich das inhaltliche Problem auf und fragt die SuS, ob sie wüssten, was Generalstände sind. Diese bejahen und beantworten die Frage:

\begin{tabular}{|c|c|}
\hline & $679[36: 44.4] 680[36: 44.8]$ \\
\hline $\begin{array}{l}\text { ToTrm8 [v] } \\
\text { OIDim8 [v] } \\
\text { Ksw [v] }\end{array}$ & $\begin{array}{l}\text { - Äh, das sind die... } \\
\text { - Das sind die, die, die, ähm, }\end{array}$ \\
\hline & $682[36: 50.3]$ \\
\hline $\begin{array}{l}\text { ToTrm8 [v] } \\
\text { OIDim8 [v] }\end{array}$ & $\begin{array}{l}\cdots \text { U Und, äh, der } \quad \text { König, der schickt die } \\
\text { verschiedenen, halt Stand drei oder sowas. } \cdots \text { Zum Beispiel... }\end{array}$ \\
\hline & $684[36: 54.5] \quad 685[36: 55.1]$ \\
\hline $\begin{array}{l}\text { ToTrm8 [v] } \\
\text { OIDim8 [v] }\end{array}$ & $\begin{array}{c}\text { dann, um, }((1,2 s)) \text { äh... } \\
\text { Um halt... }\end{array}$ \\
\hline & $686[36: 57.3] 687[36: 57.8] 688[36: 58.1]$ \\
\hline ToTrm8 [v] & ((unverständlich, $0,5 \mathrm{~s}))^{\circ}$ \\
\hline Ksw [v] & so kann man das sagen, mȟ. $((4,5 s))$ \\
\hline
\end{tabular}

Ohne auf das eigentliche Problem, nämlich die sprachliche Formulierung, wegen der die SuS ja um Hilfe gebeten hatten, einzugehen, bricht die Lehrerin die Interaktion an dieser Stelle ab: „((1s)) dann seid ihr ja gleich fertig. • - Wenn ihr überhaupt nich klar kommt könnt ihr mich nochmal rufen, aber versuchts erstmal alleine“ und verlässt die Gruppe. Die SuS der Gruppe verwenden im Anschluss die in der Erklärung für die Lehrerin gebrauchte (ungebräuchliche) und von der Lehrerin nicht korrigierte Kollokation Generalstände schicken: 
(19)

\begin{tabular}{|c|c|c|}
\hline & $698[37: 33.5] \quad 699[37: 36.8]$ & 700 [37:37.7] \\
\hline ToTrm8 [v] & Der König sch & ickt, äh, $((1,3 \mathrm{~s}))$ sol \\
\hline & $701[37: 42.6] \quad 702[37: 43.9]$ & 703 [37:45.9] \\
\hline
\end{tabular}

Auch in dieser Gruppe wird diese Formulierung in der Präsentation verwendet und nicht korrigiert. Auch hier bleibt der fachsprachliche Ausdruck den Schülern verborgen; sie erwerben ihn nicht und entwickeln kein Bewusstsein dafür, dass Fachausdrücke als solche nicht paraphrasiert werden müssen/können.

In der letzten Gruppe haben die SuS ähnliche Probleme mit dem Fachausdruck:

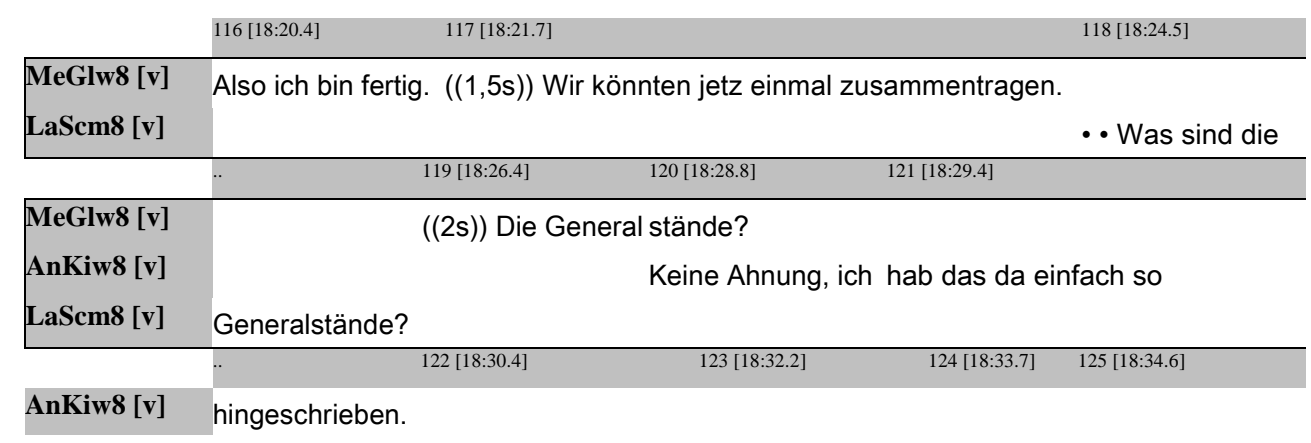

Auch hier wird die Lehrerin zu Hilfe gerufen, die das Problem inhaltlich klärt, dabei auch die fachsprachliche Kollokation Generalstände einberufen verwendet. Die Gruppe greift bis auf eine Ausnahme, in der eine Schülerin den Kollokator einrichten nutzt, auf den fachsprachlichen Kollokator zurück, unternimmt also nicht wie die anderen Gruppen den Versuch einer Paraphrase. Offen bleibt die Frage, ob sie das im Bewusstsein über die Festigkeit des Fachausdruckes tun oder sich schlicht an die Formulierung der Lehrerin bzw. des Textes anlehnen, dabei also quasi die Anforderung, eigene Worte zu verwenden, außer Acht lassen.

\section{$4 \quad$ Reflexion der Fallbeispiele und (didaktischer) Ausblick}

Die analysierten Fallbeispiele lassen den Schluss zu, dass auch Muttersprachler im Laufe ihrer schulischen Sozialisation bestimmte für den schulischen Kontext relevante Kollokationen erwerben. Es fällt auf, dass die SuS in den hier untersuchten Gruppen einer 5. und 8. Gymnasialklasse in den Gruppenarbeitsphasen am sprachlichen Ausdruck arbeiten, Formulierungsalternativen ausprobieren und dabei ein breites Spektrum an Formulierungen aufweisen. Es zeigt sich allerdings auch, dass SuS häufig zwar grammatisch inkongruente Strukturen (sensu Halliday 1985; 1988, vgl. Pohl 2007: 417) ableiten, z. B. also Nominalisierungen aus verbal realisierten Äußerungen bilden, sich dabei allerdings häufig Probleme mit dem dadurch notwendig zu verwendenden Kollokator ergeben. Dieser wird häufig gar nicht realisiert, es werden umgangssprachliche oder unangemessene Kollokatoren verwendet. Teilweise kommt es dadurch sogar zu inhaltlichen Falschdarstellungen. Es zeigt sich auch, dass der Arbeitsauftrag, eigene Worte $\mathrm{zu}$ verwenden, teils den Aufbau fachsprachlicher fester Wendungen verhindert. Hier wäre es sicher angebracht, neben einer inhaltlichen Klärung auch sprachlich mit den Schülern zu arbeiten: 
- In welchen Fällen macht es Sinn, zu paraphrasieren?

- Welche sprachlichen Mittel können für Paraphrasen genutzt werden (Synonyme, grammatische Metaphorizität (sensu Halliday 1985; 1988 , vgl. Pohl 2007: 417) etc.)?

- Welche Hilfsmittel (z. B. Kollokationswörterbücher) können dazu genutzt werden?

- In welchen sprachlichen Kontexten ist welche Formulierungsalternative angemessen, in welchen Kontexten (z. B. bei sprachlichen oder inhaltlichen Klärungsphasen in der Gruppe) sind auch eher umgangssprachliche Strukturen angemessen, in welchen sollte der fachsprachliche Ausdruck verwendet werden?

Die Beispiele zeigen, dass hier didaktisches Potential zum Teil verschenkt wird: Die SuS suchen ja gerade nach sprachlichen Formulierungen, sie arbeiten am Ausdruck und ihnen ist offensichtlich auch - so zeigen die metakognitiven Bemerkungen - bewusst, dass spezifische Formulierungsleistungen von ihnen verlangt werden. Die Analysen zeigen, dass die Schwierigkeit dabei häufig im Finden eines passenden Funktionsverbs oder Kollokators liegt, die Formulierung also von der Basis zum Kollokator aus verläuft (vgl. auch Ulrich 2016: 22). Dieser Umstand sollte in didaktische Überlegungen mit einfließen.

Der Fachunterricht bietet, so haben die Analysen gezeigt, großes Potential für die Arbeit am sprachlichen Ausdruck. Dabei geht es häufig nicht nur um ein rein sprachliches Phänomen, sondern Sprache und inhaltliches Verständnis gehen oft miteinander einher und können sich so gegenseitig stützen.

\section{Literaturverzeichnis}

Ahrenholz, Bernt (2010): „Bildungssprache im Sachunterricht der Grundschule“. In: Ahrenholz, Bernt (ed.): Fachunterricht und Deutsch als Zweitsprache. 2., durchgesehene und aktualisierte Auflage. Tübingen, Narr Verlag: 15-35.

Augst, Gerhard (demn.): „Überlegungen zur Phraseodidaktik“. In: Deutsch als Fremdsprache. Barz, Irmhild (1996): Komposition und Kollokation. In: Knobloch, Clemens/Schaeder, Burkhard (eds.): Nomination - fachsprachlich und gemeinsprachlich. Opladen, Westdeutscher Verlag: 127-146.

Beckmann, Susanne/König, Peter-Paul (2002): „Pragmatische Phraseologismen“. In: Cruse, Alan et al. (eds.): Lexikologie: Ein internationales Handbuch zur Natur und Struktur von Wörtern und Wortschätzen. Berlin/New York, de Gruyter: 421-428.

Cathomas, Rico (2007): „Neue Tendenzen in der Fremdsprachendidaktik - das Ende der kommunikativen Wende?" In: Beiträge zur Lehrerbildung 25: 180-191.

Czicza, Dániel/Hennig, Mathilde (2011): „Zur Pragmatik und Grammatik der Wissenschaftskommunikation. Ein Modellierungsvorschlag“. In: Fachsprache: international journal of specialized communication 33 (1-2): 36-60.

Dausenschön-Gay, Ulrich/Gülich, Elisabeth/Krafft, Ulrich (2007): „Vorgeformtheit als Ressource im konversationellen Formulierungs- und Verständigungsprozess“". In: Hausendorf, Heiko (ed.): Gespräch als Prozess. Linguistische Aspekte der Zeitlichkeit verbaler Interaktion. Tübingen, Gunter Narr: 181-219.

Dehn, Mechthild (2011). „Elementare Schriftkultur und Bildungssprache“. In: Sara Fürstenau \& Mechthild Gomolla (eds.): Migration und schulischer Wandel: Mehrsprachigkeit. Wiesbaden, VS Verlag für Sozialwissenschaften: 129-151. 
Eisenberg, Peter (2006): Der Satz. Grundriss der deutschen Grammatik, Bd. 2. Stuttgart/Weimar: Verlag J.B. Metzler.

Fabricius-Hansen, Cathrine (2009): „Das Verb“. In: Duden. Die Grammatik. 8., überarb. Aufl. Mannheim/Zürich, Dudenverlag: 389-566.

Feilke, Helmuth (2007): „Syntaktische Aspekte der Phraseologie II: Construction Grammar und verwandte Ansätze“. In: Phraseologie. Ein internationales Handbuch der zeitgenössischen Forschung. 1. Halbband. Berlin/New York, de Gruyter: 63-76. (= HSK Bd. 28.1).

Gülich, Elisabeth/Kotschi, Thomas (1995): "Discourse Production in Oral Communication. A Study Based on French". In: Quasthoff, Uta (ed): Aspects of Oral Communication. Berlin/New York, de Gruyter: 30-66.

Goffman, Erving (1971): Verhalten in sozialen Situationen. Gütersloh: Bertelsmann-Fachverlag.

Gogolin, Ingrid/Lange, Imke (2010): Durchgängige Sprachbildung. Eine Handreichung. Unter Mitarbeit von Dorothea Grießbach. Münster etc.: Waxmann Verlag.

Gogolin, Ingrid/Lange, Imke (2011): „Bildungssprache und Durchgängige Sprachbildung“. In: Fürstenau, Sara/Gomolla, Mechtild (eds.): Migration und schulischer Wandel: Mehrsprachigkeit. Wiesbaden, VS Verlag für Sozialwissenschaften Springer Fachmedien: 107127.

Graefen, Gabriele (2000): „Einführung in den Gebrauch der Wissenschaftssprache“. In: Wolff, Armin/Winters-Ohle, Elmar (eds.): Wie schwer ist die deutsche Sprache wirklich? Beiträge der 28. Jahrestagung DaF vom 1.-3. Juni 2000 in Dortmund. 1. Aufl. Regensburg, Fachverband Deutsch als Fremdsprache (Materialien deutsch als Fremdsprache, Heft 58): 191-210.

Grießhaber, Wilhelm (2010): „(Fach-)sprache im zweitsprachlichen Fachunterricht““. In: Ahrenholz, Bernt (ed.): Fachunterricht und Deutsch als Zweitsprache. 2., durchgesehene und aktualisierte Auflage. Tübingen, Narr Verlag: 37-53.

Gumperz, John J. (1978): “The Conversational Analysis of Interethnic Communication”. In: Ross, Ernest L. (ed.): Interethnic Communication. Athens, University of Georgia Press: $13-31$.

Gumperz, John J. (1992a):" Interviewing in intercultural situations”. In: Drew, Paul/Heritage, John (eds.): Talk at work. Interaction in institutional settings. Cambridge, Cambridge University Press: 302-327.

Gumperz, John J. (1992b): “Contextualization and understanding”. In: Duranti, Alessandro/Goodwin, Charles (eds.): Rethinking context. Cambridge, University Press: 229252.

Gumperz, John J./Cook-Gumperz, Jenny (1982): "Interethnic communication in committee negotiations". In: Gumperz, John J. (ed.): Language and Social Identity. Cambridge, Cambridge UP: $145-162$.

Häcki Buhofer, Annelies et al.(2014): Feste Wortverbindungen des Deutschen. Kollokationswörterbuch für den Alltag. Tübingen: Narr Francke Attempo.

Halliday, M. A. K. (1985): An Introduction to Functional Grammar. London et al.:Arnold.

Halliday, M. A. K. (1988): “On the language of physical science”. In: Ghadessy; Mohsen (eds.): Registers of Written English. Situational Factors and Linguistic Features. London/New York, Pinter: 162-178. 
Hausendorf, Heiko/Quasthoff, Uta (1996): Sprachentwicklung und Interaktion - eine linguistische Studie zum Erwerb von Diskursfähigkeiten. Opladen: Westdeutscher Verlag.

Hee, Katrin (i. Dr.): „Schülerkommunikation zwischen Normorientierung und pragmatischen Spielräumen“. In: Diao-Klaeger, Sabine/Albert, Georg (eds.): Mündlicher Sprachgebrauch zwischen Normorientierung und pragmatischen Spielräumen.Tübingen: Stauffenburg.

Helbig, Gerhard/Buscha, Joachim (1993): Deutsche Grammatik: Ein Handbuch für den Ausländerunterricht. Leipzig etc.: Langenscheidt.

Hoffmann, Ludger (2013): Deutsche Grammatik. Grundlagen für Lehrerausbildung, Schule, Deutsch als Zweitsprache und Deutsch als Fremdsprache. Berlin: Erich Schmidt Verlag.

Knöbl, Ralf (2011): Dialekt - Standard - Variation: Formen und Funktionen von Sprachvariation in einer mittelschwäbischen Schulklasse. Heidelberg: Universitätsverlag Winter. (OraLingua1).

Koch, Peter/Oesterreicher, Wulf (1986): „Sprache der Nähe - Sprache der Distanz. Mündlichkeit und Schriftlichkeit im Spannungsfeld von Sprachtheorie und Sprachgeschichte“. Romanistisches Jahrbuch 36: 15-43.

Köhler, Claus (1985): Verben in deutschsprachigen Fachtexten - Supplementverben (eine Voraussetzung der Normalität von Fachtextansätzen). Dresden: Technische Universität.

Köster, Lutz/Neubauer, Fritz (2002): „Kollokationen und Kompetenzbeispiele im de Gruyter Wörterbuch Deutsch als Fremdsprache“. In: Wiegand, Herbert Ernst (ed.): Perspektiven der pädagogischen Lexikographie des Deutschen II: Untersuchungen anhand des "de Gruyter Wörterbuchs Deutsch als Fremdsprachen". Tübingen, de Gruyter: 283-310.

Labov, William (1972): Language in the Inner City. Studies in the Black English Vernacular. Philadelphia: University of Pennsylvania Press.

Lange, Imke (2012): „Von ,Schülerisch` zu Bildungssprache. Übergänge zwischen Mündlichkeit und Schriftlichkeit im Konzept der Durchgängigen Sprachbildung“. In: Fürstenau, Sara (ed.): Interkulturelle Pädagogik und Sprachliche Bildung. Herausforderungen für die Lehrerbildung. Wiesbaden, Springer VS: 123-142.

Margewitsch, Erika (2005): „'Falsche“ Kollokationen und andere Formulierungsschwächen: Spuren auf dem Weg zum guten Stil?“'In: Feilke, Helmuth/Schmidlin, Regula (eds.): Literale Textentwicklung. Lang, Frankfurt am Main etc.: 185-194.

Morek, Miriam (2012): Kinder erklären. Interaktionen in Familie und Unterricht im Vergleich. Tübingen: Stauffenburg Verlag Brigitte Narr GmbH. (Stauffenburg Linguistik Bd. $60)$.

Morek, Miriam/Heller, Vivien (2012): „Bildungssprache - Kommunikative, epistemische, soziale und interaktive Aspekte ihres Gebrauchs“. In: Zeitschrift für angewandte Linguistik 57: 67-101.

Pohl, Thorsten (2007): Studien zur Ontogenese wissenschaftlichen Schreibens. Tübingen: Niemeyer.

Pohl, Thorsten (2016): „Die Epistemisierung des Unterrichtsdiskurses - ein Forschungsrahmen“. In: Bärenfänger, Olaf/Tschirner, Erwin/Möhring, Jörg (eds.): Deutsch als fremde Bildungssprache. Tübingen, Stauffenburg: 55-79.

Polenz, Peter v. (1963): Funktionsverben im heutigen Deutsch. Sprache in der rationalisierten Welt. Düsseldorf: Schwann.

Quasthoff, Uwe (2011): Wörterbuch der Kollokationen im Deutschen. Berlin/New York: de Gruyter. 
Quehl, Thomas (2010): „Die Möglichkeiten des Scaffolding. Zur Gestaltung des Übergangs von der Alltagssprache der Kinder zur Fach- und Bildungssprache“. In: Grundschulunterricht Deutsch 4: 28-32.

Reder, Anna (2006): Kollokationen in der Wortschatzarbeit. Wien: Praesens Verlag.

Rehbein, Jochen/Schmidt, Thomas/Meyer, Bernd et al. (2004): „Handbuch für das computergestützte Transkribieren nach HIAT“. In: Arbeiten zur Mehrsprachigkeit Folge B Nr. 56, Sonderforschungsbereich 538. Hamburg: Universität Hamburg.

Richert, Peggy (2005): Typische Sprachmuster der Lehrer-Schüler-Interaktion. Empirische Untersuchung zur Feedbackkomponente in der unterrichtlichen Interaktion. Bad Heilbrunn: Klinkhardt.

Roelcke, Thorsten (2002): „Fachsprache und Fachkommunikation“. In: Der Deutschunterricht 54/5: 9-20.

Spiegel, Carmen (2003): „Jugendliche diskutieren im Unterricht. Jugendsprachliche Elemente bei der Argumentationseinübung im Deutschunterricht"“. In: Neuland, Eva (ed.): Jugendsprachen - Spiegel der Zeit. Internationale Fachkonferenz 2001 an der Bergischen Universität Wuppertal. Frankfurt et al., Peter Lang: 431-445. (Sprache - Kommunikation - Kultur. Soziolinguistische Beiträge. Bd. 2).

Stein, Stefan (2003): Textgliederung. Einheitenbildung im geschriebenen und gesprochenen Deutsch Theorie und Empirie. Berlin/ New York: de Gruyter.

Steyer, Kathrin (2004): Wortverbindungen - mehr oder weniger fest. (= Jahrbuch des Instituts für Deutsche Sprache 2003). Berlin/New York: de Gruyter.

Steyer, Kathrin (2013): Usuelle Wortverbindungen. Zentrale Muster des Sprachgebrauchs aus korpusanalytischer Sicht. Tübingen: Narr.

Ulrich, Winfried (2016): Wortbildung und Kollokationen im Deutschunterricht. Förderung der Sprachkompetenz durch Erwerb morphologischer Bewusstheit, Wortschatzerweiterung und Verbesserung der Ausdrucksfähigkeit (mit 112 Arbeitsblättern in Form von Kopiervorlagen). Baltmannsweiler: Schneider Verlag Hohengehren.

Vollmer, Hemut Johannes/Thürmann, Eike (2010): ,Zur Sprachlichkeit des Fachlernens:. Modellierung eines Referenzrahmens für Deutsch als Zweitsprache“. In: Ahrenholz, Bernt (ed.): Fachunterricht und Deutsch als Zweitsprache. Tübingen, Narr: 107-132.

Wallner, Franziska (2014): Kollokationen in Wissenschaftssprachen. Zur lernerlexikographischen Relevanz ihrer wissenschaftssprachlichen Gebrauchsspezifika. Tübingen: Narr.

Wieler, Petra (2011): „'Denn sie erkannten nicht die Gefahr ${ }^{\text {- }}$ bildungssprachliche Aspekte in Gesprächen und Texten von Kindern im Deutschunterricht der Grundschule und darüber hinaus“". In: Petra Hüttis-Graf/Wieler, Petra (eds.): Übergänge zwischen Mündlichkeit und Schriftlichkeit im Vor- und Grundschulalter. Freiburg, Fillibach: 123-149.

Zifonun, Gisela/Hoffmann, Ludger/ Strecker, Bruno (1997): Grammatik der deutschen Sprache, Bd.1. Berlin/New York, de Gruyter: 703-705

Zifonun, Gisela/Hoffmann, Ludger/ Strecker, Bruno (1997): Grammatik der deutschen Sprache, Bd.2. Berlin/New York, de Gruyter: 1068-1071.

\section{Einbezogene Primärliteratur}

Regenhardt, Hans-Otto (eds..) (2008): Forum Geschichte 5/6. Niedersachsen. Von der Vorgeschichte zum frühen Mittelalter. Berlin: Cornelsen: 78-79.

Regenhardt, Hans-Otto (eds..) (2009): Forum Geschichte 8. Niedersachsen. Vom Absolutismus 
bis zur Industrialisierung. Berlin: Cornelsen: 49.

\section{Anhang:}

\section{Das Niltal wird besiedelt}

Leben nach den Regeln des Flusses?

Als vor etwa 9000 Jahren die Warmzeit begann, trockneten große Teile Nordafrikas allmählich aus und wurden zur Wuiste. Einige Menschen passten sich den erschwerten Bedingungen an und lebten mit ihren Viehherden als Nomaden in diesen kargen Gebieten. Jedoch die meisten Menschen verließen die Wüste und bießen sich als Bauern an den wasserreichen Ufern des Nils nieder.

Whrend der Regenzeit im juni stròmten aus Afrikas tropischen Gebieten ungeheure Wassermassen in den Nil. In dieser Zeit stieg der Wasserstand um 5 bis $7 \mathrm{Me}$ ter. Ende September, wenn der Nil in sein Flussbett zuruckgekehut war, blieb zu beiden Seiten des Ufers eine Schlarnmschicht zuruck. Die Menschen erkannten schnell, dass diese Schlammschicht sehr fruchtbar war und sich besonders gut als Ackerboden eignete. Sie pflugten und wasserten die Ácker, satten Getreide und bauten vielerlei Pflanzen an. Vier Monate spalter konnten das Korn geschnitten, Linsen, Bohnen, Trauben, Datteln und Feigen geerntet werden. Danach lag das Land vier Monate lang brach.

Die Hohe der Flut entschied aber ,fette" und _magere* Jahre. Oft machte eine zu grofe Oberschwemmung die Ernte zunichte, eine zu geringe hatte eine Hungersnot zur Folge. So konnte der Nil Segen oder Fluch sein.

Um sich von den Unwaggbarkeiten des Nils unabhăngiger zu machen, mussten die Menschen Deiche, Kanale und Staudimme basen. Dazu war es notwendig, dass die Ägypter ihr Zusammenleben genau organisierten und die Arbeit untereinander aufteilten.

Oberschwernmungen fuhrten dazu, dass die Felder wieder neu vermessen werden mussten. Als natioliche Maßeinheiten verwandten die Agypter Ele und Fuß. Aus der Feldvermessung entwickelte sich eine Wissen. schaft, die von den Griechen später Geometrie genannt wurde.

Im Laufe der Zeit entdeckten die Ágypter, dass es von einer Niluberschwemmung bis zur năchsten 365 Tage dauerte. Aus dieser Erkenntnis entwickelten sie einen Kalender, der im Prinzip noch heute Gultigkeit hat.

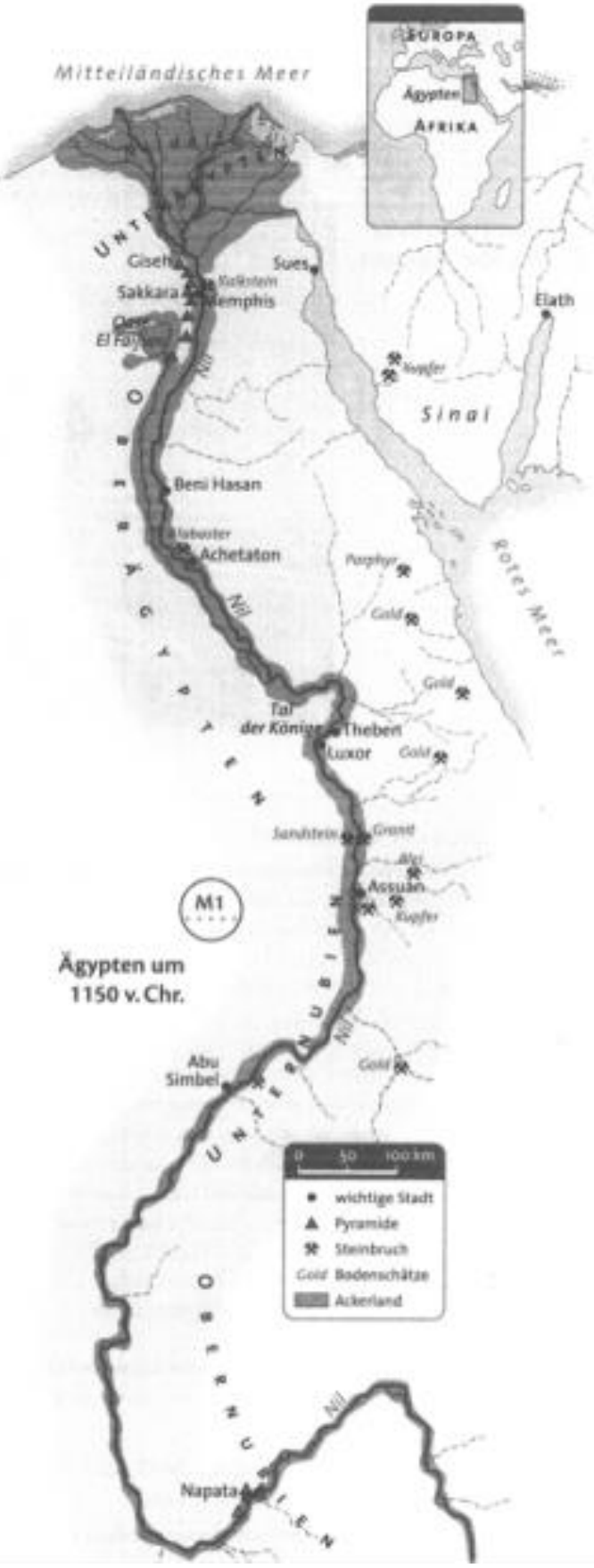

Regenhardt, Hans-Otto (Hrsg.) (2008: 78) 


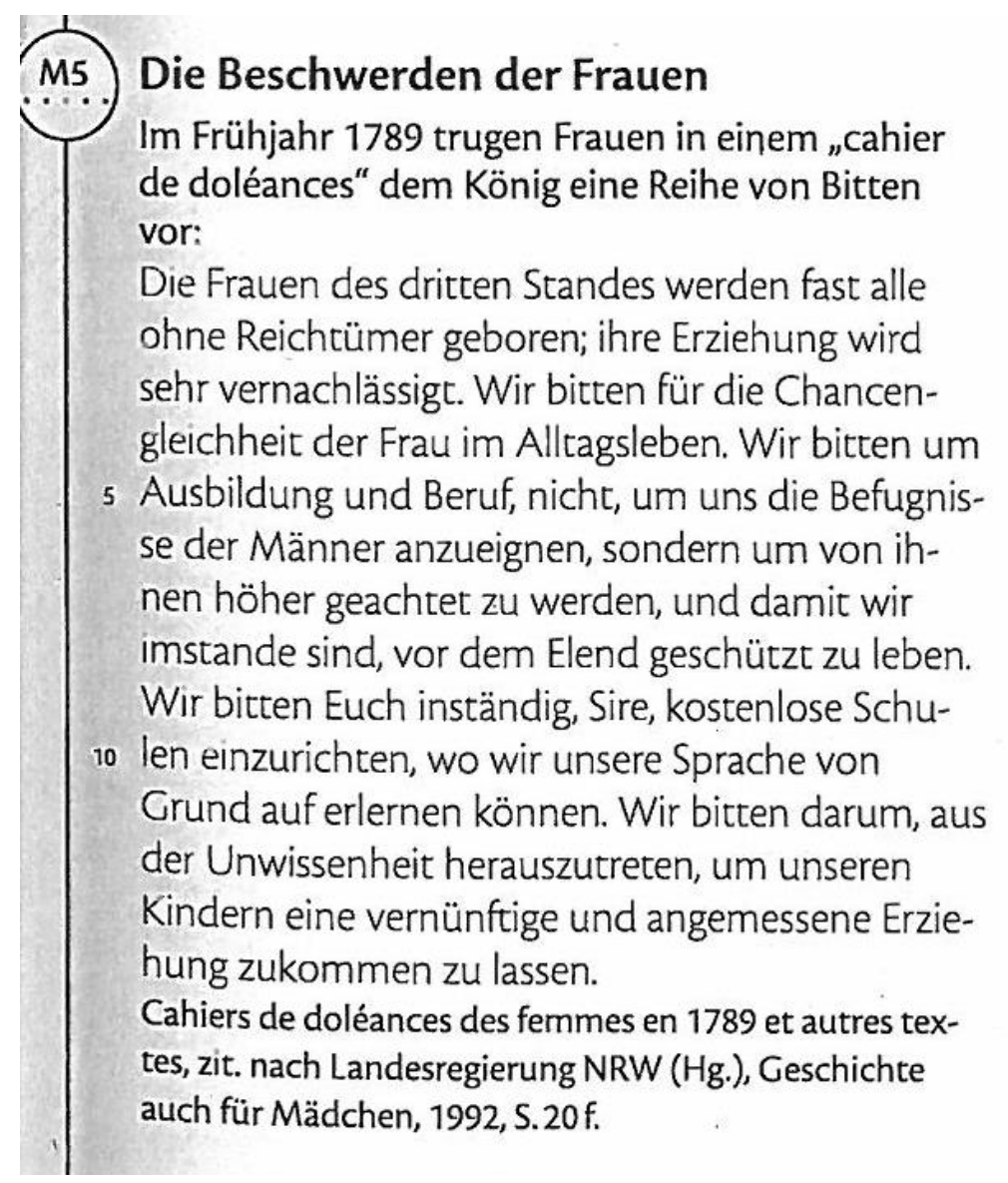

Regenhardt, Hans-Otto (Hrsg.) (2009: 49) 


\footnotetext{
1

Die Beschwerden der Bauern

Das Beschwerdeheft der Gemeinde Letteguives vom 29. März 1789, ein Beispiel für die, „cahiers de doléances" der Bauern:

2. Diese Großbauern werden auch Herren der Lebensmittelpreise und des Schicksals der Allgemeinheic und verkaufen ihr Getreide erst, wenn sie es mehrere Jahre aufbewahrs haben, und sie

s halten es sogar zurück, wenn sie können ... Der hohe Preis isc die Ursache der Zerstönung des ganzen Handels, weil man keine andere Ware mehr kaufen kann, nachdem man Bror gekauft hat ... Andere ... sterben an Hunger...

a 8. Möge der König vor dem Bankrott bewahren, der den Handel noch ruiniert...

14. Dass die Salzsteuer abgeschafft werde und der Verkauf von Salz frei sei...

16. Die drei Stände mōgen dem: König im Ver-

is hälmis zu ihren Einkünften zahlen...

18. Der König wird demütig gebeten die Generalstănde alle fünf Jahre einzuberufen, um von unserem Unglück zu erfahren.

Zit nach Wolf Dietrich Behschnitt, Die Französische Rewolution, Stuttgart (Klett) 1983, S. 30.
}

Regenhardt, Hans-Otto (Hrsg.) (2009: 49) 